\title{
Robust global recurrence for a class of stochastic hybrid systems
}

\author{
Anantharaman Subbaraman*, Andrew R. Teel* \\ Robert Bosch Research and Technology Center, Palo Alto, CA 94304 \\ Department of Electrical and Computer Engineering, University of California, Santa Barbara, CA 93106
}

\begin{abstract}
We study a weak property called recurrence for a class of stochastic hybrid systems and establish robustness of the recurrence property. In particular, we establish that recurrence of an open, bounded set is robust to sufficiently small perturbations in the set, perturbations of the data of the stochastic hybrid system and modifications to the system data that slow down the recurrence property. The robustness results are a consequence of the mild regularity properties assumed for the stochastic hybrid system.
\end{abstract}

Keywords: Stochastic hybrid systems; Robust stability; Uniform global recurrence.

\section{Introduction}

Stochastic hybrid systems (SHS) combine continuous-time evolution, discrete-time events and probabilistic behavior. Frameworks for modeling SHS are in [1], [2], [3] and [4]. SHS models arise frequently in the context of complex systems like air traffic management systems, networked control systems and systems biology. See [5], [6] and [7] for more details. The recent survey paper [8] presents a unified modeling framework for the various SHS representations in the literature and addresses stability related issues. In particular, important topics that are well studied in the case of non-stochastic hybrid systems, like sufficient conditions for stability, weak sufficient conditions for stability, invariance principle, robust stability conditions and converse Lyapunov theorems, are analyzed in detail in [8] for stochastic hybrid systems.

In this paper, we address one aspect of the future research directions proposed in [8]. We study a weak stochastic property called recyrrence and establish robustness of recurrence to various state dependent perturbations. The class of systems for which we study this property are stochastic hybrid systems with randomness restricted to the discrete-time dynamics. The system model we study can account for spontaneous transitions, forced transitions and probabilistic resets. While there are many interesting developments in the area of stochastic differential inclusions, the results needed to establish robustness particularly sequential compactness of solutions with mild regularity assumptions on the system data are not available yet and hence a more general study of such systems is a challenge.

*Research supported in part by AFOSR FA9550-12-1-0127, NSF ECCS-1508757, AFOSR FA9550-15-1-0155 and NSF ECCS1232035.

Email addresses: anantharaman.subbaraman@us.bosch.com (Anantharaman Subbaraman), teel@ece.ucsb.edu (Andrew R. Teel)

Preprint submitted to Nonlinear Analysis: Hybrid Systems

July 29, 2016

(C) 2016. This manuscript version is made available under the Elsevier user license

http://www.elsevier.com/open-access/userlicense/1.0/ 
We adopt the framework for modeling SHS with non-unique solutions proposed in [2] and [9]. This class of systems covers other frameworks such as piecewise-deterministic Markov processes (PDMP) and Markov jump systems. We study stochastic systems with non-unique solutions for two key reasons. Firstly, analyzing such systems is crucial to developing a robust stability theory. Robust stability for non-stochastic hybrid systems is studied in [10]. Robustness of the stability property is also crucial in developing converse Lyapunov theorems (see [11, 12]). Secondly, such system models allow flexibility in control design applications (see [13], [14] and [15]).

The stochastic property of interest in this paper is called recurrence. Loosely speaking, recurrence of an open set implies that solutions visit the set infinitely often with probability one. The recurrence property is frequently studied in the literature on stochastic systems. See [1] and [16]. Recurrence is a weaker notion of stability compared to more commonly studied notions of mean square asymptotic stability and asymptotic stability in probability. Generally, recurrence of a set need not imply any stability-like property or invariance-like property for the set. In fact, recurrence of the set does not necessarily imply solutions stay bounded in a probabilistic sense. Still, recurrence is useful to study in situations where stronger properties like convergence or asymptotic stability cannot be established. See [17] for an example in the context of source-seeking by a MAV (Micro Aerial Vehicle) where persistent disturbances prevent establishing stronger properties. Recurrence can also be used in situations to provide a sharper characterization for the behavior of solutions even though asymptotic stability properties may be satisfied by certain sets. See [12, Example 1] for more details.

The recurrence property when studied for non-stochastic systems with respect to bounded sets is equivalent to the well studied property of ultimate boundedness. The recurrence property is studied with respect to open sets because for closed sets recurrence is generally not equivalent to uniform recurrence and need not be robust. See [12] for more details. The equivalence between recurrence of bounded sets and ultimate boundedness of solutions does not hold in general for stochastic systems.

Robustness of stability can be loosely defined as the stability property for the nominal system being preserved when the system is affected by sufficiently small perturbations. In [18 results on robustness of recurrence for a class of stochastic hybrid systems are explored. The robustness results in [18] exploit the regularity properties of the nominal system. In this work, we provide detailed proofs and examples highlighting the main results established in [18. The results proved in this paper can be used to establish a converse Lyapunov theorem for recurrence in stochastic hybrid systems.

The rest of the paper is organized as follows. Section 2 presents the basic notation and definitions to be used in the paper. Section 3 introduces the SHS framework that will be considered in the rest of the paper. The recurrence property is explained in Section 4. Section 5 introduces viability and reachability probabilities which will be used to prove the main results of the paper. Section 6 establishes some basic bounds related to these probabilities. The main results related to robustness of the recurrence property to various state dependent perturbations are presented in Section 7. The proof of the main results in the paper 
rely on the properties of viability and reachability probabilities introduced in [2] and the results on sequential compactness of solutions for a class of stochastic hybrid systems established in [19]. Section 8 includes a short discussion of the application of the robustness results in developing a converse Lyapunov theorem for recurrence. Section 9 presents some concluding comments. The appendix contains proofs of the results from Sections 6-7.

\section{Basic notation and definitions}

For a closed set $S \subset \mathbb{R}^{n}$ and $x \in \mathbb{R}^{n},|x|_{S}:=\inf _{y \in S}|x-y|$ is the Euclidean distance of $x$ to $S$. $\mathbb{B}$ (resp., $\mathbb{B}^{o}$ ) denotes the closed (resp., open) unit ball in $\mathbb{R}^{n}$. Given a closed set $S \subset \mathbb{R}^{n}$ and $\epsilon>0, S+\epsilon \mathbb{B}$ (resp., $S+\epsilon \mathbb{B}^{o}$ ) represents the set $\left\{x \in \mathbb{R}^{n}:|x|_{S} \leq \epsilon\right\}$ (resp., $\left\{x \in \mathbb{R}^{n}:|x|_{S}<\epsilon\right\}$ ). $\mathbb{R}_{\geq 0}$ denotes the non-negative real numbers; $\mathbb{Z}_{\geq 0}$ denotes the non-negative integers. Let $\mathcal{T}$ be a topological space. A function $\Psi: \mathcal{T} \rightarrow \mathbb{R}_{\geq 0}$ is upper semicontinuous if for every converging sequence $\left\{t_{i}\right\} \rightarrow t, \limsup _{i \rightarrow \infty} \Psi\left(t_{i}\right) \leq \Psi(t)$. For $S \subset \mathbb{R}^{n}$, the symbol $\mathbb{I}_{S}$ denotes the indicator function of $S$ i.e., $\mathbb{I}_{S}(x)=1$ for $x \in S$ and $\mathbb{I}_{S}(x)=0$ otherwise. Following [2], we define for sets $S_{1}, S_{2} \subset \mathbb{R}^{n}, \mathbb{I}_{\subset S_{1}}\left(S_{2}\right)=1-\sup _{x \in S_{2}} \mathbb{I}_{\mathbb{R}^{n} \backslash S_{1}}(x)$ and $\mathbb{I}_{\cap S_{1}}\left(S_{2}\right)=$ $\sup _{x \in S_{2}} \mathbb{I}_{S_{1}}(x)$ with the convention that the maximum's are zero when $S_{2}=\varnothing$. For $\tau \geq 0$, we define the set $\Gamma_{\leq \tau}:=\left\{(s, t) \in \mathbb{R}^{2}: s+t \leq \tau\right\}$. The sets $\Gamma_{<\tau}, \Gamma_{\geq \tau}$ are defined similarly. A set-valued mapping $M: \mathbb{R}^{p} \rightrightarrows \mathbb{R}^{n}$ is outer semicontinuous if, for each $\left(x_{i}, y_{i}\right) \rightarrow(x, y) \in \mathbb{R}^{p} \times \mathbb{R}^{n}$ satisfying $y_{i} \in M\left(x_{i}\right)$ for all $i \in \mathbb{Z}_{\geq 0}, y \in M(x)$. A mapping $M$ is locally bounded if, for each bounded set $K \subset \mathbb{R}^{p}, M(K):=\bigcup_{x \in K} M(x)$ is bounded. $\mathbf{B}\left(\mathbb{R}^{m}\right)$ denotes the Borel $\sigma$-field. A set $F \subset \mathbb{R}^{m}$ is measurable if $F \in \mathbf{B}\left(\mathbb{R}^{m}\right)$. For a measurable space $(\Omega, \mathcal{F})$, a mapping $M: \Omega \rightrightarrows \mathbb{R}^{n}$ is $\mathcal{F}$-measurable [20, Def. 14.1] if, for each open set $\mathcal{O} \subset \mathbb{R}^{n}$, the set $M^{-1}(\mathcal{O}):=\{\omega \in \Omega: M(\omega) \cap \mathcal{O} \neq \varnothing\} \in \mathcal{F}$. The functions $\pi_{i}: \mathbb{R}_{\geq 0} \times \mathbb{R}_{\geq 0} \times \mathbb{R}^{n} \rightarrow \mathbb{R}_{\geq 0}$ are such that $\pi_{i}\left(t_{1}, t_{2}, z\right)=t_{i}$ for each $i \in\{1,2\}$. A function $\kappa: \mathbb{R}_{\geq 0} \rightarrow \mathbb{R}_{\geq 0}$ belongs to class- $\mathcal{K}$ if it is continuous, strictly increasing and $\kappa(0)=0$. A function $\kappa: \mathbb{R}_{\geq 0} \rightarrow \mathbb{R}_{\geq 0}$ belongs to class- $\mathcal{K}_{\infty}$ it if belongs to class- $\mathcal{K}$ and is unbounded.

\section{Preliminaries on stochastic hybrid systems}

We consider a class of SHS studied in [2]. Let the state $x \in \mathbb{R}^{n}$ and random input $v \in \mathbb{R}^{m}$. Then, the SHS is written formally as

$$
\begin{aligned}
\dot{x} & \in F(x), \quad x \in C \\
x^{+} & \in G\left(x, v^{+}\right), \quad x \in D \\
v & \sim \mu(\cdot)
\end{aligned}
$$

where $C, D \subset \mathbb{R}^{n}$ represent the flow and jump sets respectively and $F: \mathbb{R}^{n} \rightrightarrows \mathbb{R}^{n}, G: \mathbb{R}^{n} \times \mathbb{R}^{m} \rightrightarrows \mathbb{R}^{n}$ represent the flow and jump maps respectively. So, the continuous-time dynamics are modeled by a differential inclusion and the discrete-time dynamics are modeled by a stochastic difference inclusion. 
The distribution function $\mu$ is derived from the probability space $(\Omega, \mathcal{F}, \mathbb{P})$ and a sequence of independent, identically distributed (i.i.d.) input random variables $\mathbf{v}_{i}: \Omega \rightarrow \mathbb{R}^{m}$ defined on $(\Omega, \mathcal{F}, \mathbb{P})$ for $i \in \mathbb{Z}_{\geq 1}$. Then $\mu$ is defined as $\mu(A)=\mathbb{P}\left(\omega \in \Omega: \mathbf{v}_{i}(\omega) \in A\right)$ for every $A \in \mathbf{B}\left(\mathbb{R}^{m}\right)$. We denote by $\mathcal{F}_{i}$ the collection of sets $\left\{\omega:\left(\mathbf{v}_{1}(\omega), \ldots, \mathbf{v}_{i}(\omega)\right) \in A\right\}, A \in \mathbf{B}\left(\left(\mathbb{R}^{m}\right)^{i}\right)$ which are the sub- $\sigma$ fields of $\mathcal{F}$ that form the natural filtration of $\mathbf{v}=\left\{\mathbf{v}_{i}\right\}_{i=1}^{\infty}$. For simplicity we will refer the stochastic hybrid system (1) through its data as

$$
\mathcal{H}=(C, F, D, G, \mu)
$$

We now state the basic assumptions imposed throughout the rest of the paper on the data of the SHS $\mathcal{H}$.

Standing Assumption 1. The data of the SHS $\mathcal{H}$ satisfies the following conditions:

1. The sets $C, D \subset \mathbb{R}^{n}$ are closed;

2. The mapping $F: \mathbb{R}^{n} \rightrightarrows \mathbb{R}^{n}$ is outer-semicontinuous, locally bounded with nonempty convex values on $C$;

3. The mapping $G: \mathbb{R}^{n} \times \mathbb{R}^{m} \rightrightarrows \mathbb{R}^{n}$ is locally bounded and the mapping $v \mapsto \operatorname{graph}(G(\cdot, v)):=\{(x, y) \in$ $\left.\mathbb{R}^{2 n}: y \in G(x, v)\right\}$ is measurable with closed values.

We now explain the motivation for Standing Assumption 1. Firstly, the equivalence between uniform and non-uniform versions of recurrence for open, bounded sets exploit the regularity properties. Secondly, for the recurrence property to be robust to sufficiently small perturbations we need the system properties in Standing Assumption 1. Finally, it guarantees the existence of random solutions for the SHS. We refer the reader to 2 for more details. If the flow map is a function then it satisfies item 2 of Standing Assumption 1 if and only if it is continuous; see [20, Corollary 5.20]. If the jump map is a function then it satisfies item 3 of Standing Assumption 1 if and only if it is locally bounded and satisfies the so-called Caratheodory conditions, namely, $x \mapsto g(x, v)$ is continuous for each $v$ and $v \mapsto g(x, v)$ is measurable for each $x$; see [20, Example 14.15] for the sufficiency of these conditions, and for necessity note that continuity of $x \mapsto g(x, v)$ follows from the values of $v \mapsto \operatorname{graph}(G(., v))$ being closed and [20, Corollary 5.20] while measurability of $v \mapsto g(x, v)$ follows from measurability of $v \mapsto \operatorname{graph}(G(., v))$ and [20, Prop. 14.11(a)] with $S_{1}(v):=\operatorname{graph}(G(. v))$ and $S_{2}(v):=\{x\} \times \mathbb{R}^{n}$.

We note that the conditions in Standing Assumption 1 are an extension of the conditions used in [10] for non-stochastic hybrid systems modeled by set-valued maps.

We now explain the notion of a solution to non-stochastic hybrid systems studied in [10]. A compact hybrid time domain is a subset of $\mathbb{R}_{\geq 0} \times \mathbb{Z}_{\geq 0}$ of the form $\cup_{j=0}^{J}\left(\left[t_{j}, t_{j+1}\right] \times\{j\}\right)$ for some $J \in \mathbb{Z}_{\geq 0}$ and real numbers $0=t_{0} \leq t_{1} \ldots \leq t_{J+1}$. A hybrid time domain is a set $E \subset \mathbb{R}_{\geq 0} \times \mathbb{Z}_{\geq 0}$ such that for each $T, J$, the set $E \cap([0, T] \times\{0, \ldots, J\})$ is a compact hybrid time domain. A hybrid arc is a mapping $\phi: E \rightarrow \mathbb{R}^{n}$ such that $E$ is a hybrid time domain and for each $j \in \mathbb{Z}_{\geq 0}$, the mapping $t \mapsto \phi(t, j)$ is locally absolutely continuous. The system (1a) is said to have no finite escape times if there are no solutions of 1a that escape to infinity at a finite time. 
Let $(\Omega, \mathcal{F})$ be a measurable space. A stochastic hybrid arc is a mapping $\mathbf{x}$ defined on $\Omega$ such that $\mathbf{x}(\omega)$ is a hybrid arc for each $\omega \in \Omega$ and the set-valued mapping from $\Omega$ to $\mathbb{R}^{n+2}$ defined by

$$
\omega \mapsto \operatorname{graph}(\mathbf{x}(\omega)):=\{(t, j, z): \phi=\mathbf{x}(\omega),(t, j) \in \operatorname{dom}(\phi), z=\phi(t, j)\}
$$

is $\mathcal{F}$-measurable with closed values. Define $\operatorname{graph}(\mathbf{x}(\omega))_{\leq j}:=\operatorname{graph}(\mathbf{x}(\omega)) \cap\left(\mathbb{R}_{\geq 0} \times\{0, \ldots, j\} \times \mathbb{R}^{n}\right)$. An $\left\{\mathcal{F}_{j}\right\}_{j=0}^{\infty}$ adapted stochastic hybrid arc is a stochastic hybrid arc $\mathbf{x}$ such that the mapping $\omega \mapsto \operatorname{graph}(\mathbf{x}(\omega))_{\leq j}$ is $\mathcal{F}_{j}$-measurable for each $j \in \mathbb{Z}_{\geq 0}$. An adapted stochastic hybrid $\operatorname{arc} \mathbf{x}$ is a solution starting from $x$ if $\mathbf{x}(\omega)$ is a solution to $\mathcal{H}$ with inputs $\left\{\mathbf{v}_{i}(\omega)\right\}_{i=1}^{\infty}$; that is with $\phi_{\omega}:=\mathbf{x}(\omega)$ we have

- $\phi_{\omega}(0,0)=x$

- if $\left(t_{1}, j\right),\left(t_{2}, j\right) \in \operatorname{dom}\left(\phi_{\omega}\right)$ with $t_{1}<t_{2}$ then, for almost every $t \in\left[t_{1}, t_{2}\right], \phi_{\omega}(t, j) \in C$ and $\dot{\phi}_{\omega}(t, j) \in$ $F\left(\phi_{\omega}(t, j)\right)$

- if $(t, j),(t, j+1) \in \operatorname{dom}\left(\phi_{\omega}\right)$ then $\phi_{\omega}(t, j) \in D$ and $\phi_{\omega}(t, j+1) \in G\left(\phi_{\omega}(t, j), \mathbf{v}_{j+1}(\omega)\right)$.

We refer the reader to 2 for more details on the solution concept to SHS. In this paper, we will focus on random solutions that are (pathwise) maximal. Loosely speaking, a random solution is (pathwise) maximal if for each sample path the domain of the sample path cannot be extended further. See [10, Def 2.7] for more details. A random solution is almost surely complete if for almost every $\omega$, the sample path $\mathbf{x}(\omega)$ is complete. A sample path is said to be complete if the domain of the sample path is unbounded. See [10, Def 2.5].

\section{Recurrence and Uniform recurrence}

We now define the notion of recurrence for open, bounded sets. We denote by $\mathcal{S}_{r}(x)$, the set of random solutions generated by $\mathcal{H}$ from the initial condition $x$.

Definition 1. An open, bounded set $\mathcal{O} \subset \mathbb{R}^{n}$ is globally recurrent for $\mathcal{H}$ if there are no finite escape times for 11a and for each $x \in \mathbb{R}^{n}$ and $\mathbf{x} \in \mathcal{S}_{r}(x)$,

$$
\lim _{\tau \rightarrow \infty} \mathbb{P}\left(\left(\operatorname{graph}(\mathbf{x}) \subset\left(\Gamma_{<\tau} \times \mathbb{R}^{n}\right)\right) \vee\left(\operatorname{graph}(\mathbf{x}) \cap\left(\Gamma_{\leq \tau} \times \mathcal{O}\right) \neq \varnothing\right)\right)=1
$$

Loosely speaking, the above condition insists that almost surely the sample paths of the random solution $\mathbf{x}$ are either not complete or hit the set $\mathcal{O}$.

For each $x \in \mathbb{R}^{n}$ and $\mathbf{x} \in \mathcal{S}_{r}(x)$, define $\Omega_{1}^{\mathbf{x}}:=\left\{\omega: \exists(t, j) \in \operatorname{dom}(\mathbf{x}(\omega))\right.$ such that $\left.\mathbf{x}_{\omega}(t, j) \in \mathcal{O}\right\}$, $\Omega_{2}^{\mathbf{x}}=\left\{\omega: \exists \tau>0\right.$ such that $\left.\operatorname{graph}(\mathbf{x}(\omega)) \subset\left(\Gamma_{<\tau} \times \mathbb{R}^{n}\right)\right\}$. Consequently, an open, bounded set $\mathcal{O} \subset \mathbb{R}^{n}$ is globally recurrent for $\mathcal{H}$ if there are no finite escape times for 1 a and for each $x \in \mathbb{R}^{n}$ and $\mathbf{x} \in \mathcal{S}_{r}(x)$, $\mathbb{P}\left(\Omega_{1}^{\mathbf{x}} \cup \Omega_{2}^{\mathbf{x}}\right)=1$.

Example 1: Consider a SHS with the system dynamics

$$
\begin{aligned}
\dot{x} & =f(x), x \in C \\
x^{+} & =g(x, v), x \in D
\end{aligned}
$$


where $f(x)=1, g(x, v)=v x$ with $v \in\{0,1\}, \mu(\{0\})=\mu(\{1\})=0.5, C=(-\infty, 1]$ and $D=[1,2]$. For this system, it can be observed that any set of the form $\mathcal{O}=(-\epsilon, \epsilon)$ with $0<\epsilon<1$ is globally recurrent. For any initial condition $x_{0} \in C$ such that $x_{0} \in(-\infty, \epsilon)$, solutions hit the set $\mathcal{O}$ due to the continuous-time dynamics. For initial conditions $x_{0} \in C$ such that $x_{0} \geq \epsilon$, the solutions reach the set $D$. Then, for solutions from the set $D$, almost surely the random input $v=0$ appears in a sequence of random inputs $\left\{v_{i}\right\}_{i=0}^{\infty}$. Hence the solutions from the set $D$ reaches the origin almost surely. This establishes global recurrence of the set $\mathcal{O}$. We can easily observe from this example that the set $\mathcal{O}$ is not invariant in a probabilistic sense (loosely speaking, a set is invariant in the probabilistic sense if solutions starting from the set stay in the set in an almost sure sense. The concept of probabilistic invariance is discussed more in [21] and [22]), as the continuous-time dynamics ensures that solutions leave the set $\mathcal{O}$ and reach $D$. Similarly, we can observe that the set $\mathcal{O}$ does not have any stability-like property.

Definition 2. An open, bounded set $\mathcal{O} \subset \mathbb{R}^{n}$ is uniformly globally recurrent for $\mathcal{H}$ if there are no finite escape times for (1a) and for each $\rho>0$ and compact set $K$ there exists $\tau \geq 0$ such that for $\xi \in K$ and $\mathbf{x} \in \mathcal{S}_{r}(\xi)$,

$$
\mathbb{P}\left(\left(\operatorname{graph}(\mathbf{x}) \subset\left(\Gamma_{<\tau} \times \mathbb{R}^{n}\right)\right) \vee\left(\operatorname{graph}(\mathbf{x}) \cap\left(\Gamma_{\leq \tau} \times \mathcal{O}\right) \neq \varnothing\right)\right) \geq 1-\rho .
$$

Example 2: Consider the SHS in Example 1. We are now going to establish that the set $\mathcal{O}$ is uniformly globally recurrent. Let a compact set $K$ and $\rho>0$ in the definition of uniform global recurrence be given. Choose $\tau^{*} \in \mathbb{Z}_{\geq 0}$ such that $1-(0.5)^{\tau^{*}} \geq 1-\rho$. We first consider the case when the set $K \subset D$. In this case, we can choose $\tau \geq \tau^{*}$. If the compact set $K \subset[\epsilon, 1]$, the time $\tau$ in the definition of uniform global recurrence is chosen such that $\tau \geq(1-\epsilon)+\tau^{*}$. If the compact set $K \subset(-\epsilon, \epsilon)$, we can choose $\tau=0$. Similarly, if the compact set $K \subset(-\infty,-\epsilon]$, the time $\tau$ can be chosen such that $\tau \geq \max _{x \in K}(|x|-\epsilon)+1$. The choice of $\tau$ for any other compact set $K$ can be derived from the above cases by taking the maximum of $\tau$ among the above cases considered for the set $K$.

The following result establishes equivalence between uniform and non-uniform recurrence. See [19, Thm 6] for a proof (Cf. Proposition 2).

Proposition 1. An open, bounded set $\mathcal{O}$ is globally recurrent for $\mathcal{H}$ if and only if it is uniformly globally recurrent for $\mathcal{H}$.

As explained in 12, the recurrence property is generally studied with respect to open sets for two reasons. Firstly, for closed sets recurrence and uniform recurrence are not necessarily equivalent. Secondly, for closed sets the recurrence property need not be robust. See [12, Example 3, 5] for more details.

\section{Viability and reachability probabilities}

It can be observed from the definition of global recurrence that the recurrence property needs to hold for every random solution generated from any initial condition. Hence, it is useful to work with the probabilities 
that reflect the behavior of all random solutions generated from any initial condition. As in [23] and [2] we characterize the recurrence property in terms of viability probabilities defined below.

For $x \in \mathbb{R}^{n}, \tau \geq 0$ and closed set $S \subset \mathbb{R}^{n}$, we define

$$
m_{\subset S}(\tau, x):=\sup _{\mathbf{x} \in \mathcal{S}_{r}(x)} \mathbb{P}\left(\left(\operatorname{graph}(\mathbf{x}) \cap\left(\Gamma_{\geq \tau} \times \mathbb{R}^{n}\right) \neq \varnothing\right) \wedge\left(\operatorname{graph}(\mathbf{x}) \cap\left(\Gamma_{\leq \tau} \times \mathbb{R}^{n}\right) \subset \mathbb{R}^{2} \times S\right)\right)
$$

The viability probability $m_{\subset S}(\tau, x)$ is related to the largest probability that random solutions starting from $x$ stay in the set $S$ for hybrid time less than or equal to $\tau$ and not stop before that time. This probability condition is complementary to the condition for recurrence when the set $S=\mathbb{R}^{n} \backslash \mathcal{O}$ and when $\tau \rightarrow \infty$.

It is established in [2, Prop 10.2, Prop 9.1] that the supremum in the above definition is achieved for some random solution and the mapping $(\tau, x) \mapsto m_{\subset S}(\tau, x)$ is upper semicontinuous. The conditions needed to achieve the supremum in the definition are the conditions in Standing Assumption 1 and the absence of finite escape times for the solutions of the continuous-time dynamics. Since the systems studied in this paper have certain open, bounded sets recurrent, the conditions needed for [2, Prop 10.2, Prop 9.1] are satisfied. We refer the reader to [2, Section 9] for more details. Define

$$
\widehat{m}_{\subset S}(x):=\lim _{\tau \rightarrow \infty} m_{\subset S}(\tau, x)
$$

The quantity $\widehat{m}_{\subset S}(x)$ is related to the infinite time viability probability. The limit is well defined due to the mapping $\tau \mapsto m_{\subset S}(\tau, x)$ being non-increasing for every $x$ and lower bounded by zero.

The following proposition proved in the appendix holds for any SHS satisfying Standing Assumption 1 and provides an equivalent characterization for global recurrence. Roughly, recurrence of a set $\mathcal{O}$ implies that solutions keep returning to the set $\mathcal{O}$ infinitely often with probability one. This implies that solutions cannot stay in the complement of the set $\mathcal{O}$ for all time and hence, in essence, the set $\mathbb{R}^{n} \backslash \mathcal{O}$ is not viable.

Proposition 2. Let $\mathcal{O} \subset \mathbb{R}^{n}$ be an open, bounded set. The following statements are equivalent:

1. $\mathcal{O}$ is globally recurrent.

2. $\widehat{m}_{\subset \mathbb{R}^{n} \backslash \mathcal{O}}(x)=0$ for all $x \in \mathbb{R}^{n}$.

3. For every compact set $K \subset \mathbb{R}^{n}$, and $\rho>0$, there exists $\tau \geq 0$ such that $\sup _{x \in K} m_{\subset \mathbb{R}^{n} \backslash \mathcal{O}}(\tau, x) \leq \rho$.

We also utilize reachability probabilities studied in [2, Section 8]. For $x \in \mathbb{R}^{n}, \tau \geq 0$ and closed set $S \subset \mathbb{R}^{n}$, we define

$$
m_{\cap S}(\tau, x):=\sup _{\mathbf{x} \in \mathcal{S}_{r}(x)} \mathbb{P}\left(\operatorname{graph}(\mathbf{x}) \cap\left(\Gamma_{\leq \tau} \times S\right) \neq \varnothing\right)
$$

The reachability probability $m_{\cap S}(\tau, x)$ is related to the largest probability that random solutions starting from $x$ reach the set $S$ within a time bound (combination of continuous-time and discrete time) $\tau$. It can be established similar to [2, Prop 10.2] that the supremum in the above definition is achieved for some random solution. In [24] and 25] computational techniques related to reachability probabilities are explored in more detail. The reachability and viability probabilities are important tools for establishing robustness 
results for stochastic systems. A similar approach is explored in [26] to establish robustness results for a class of stochastic difference inclusions. In the next section, results that relate the viability and reachability probabilities similar to [26] are established for a class of stochastic hybrid systems.

\section{Preliminary bounds on viability and reachability probabilities}

In this section, we focus on a SHS $\tilde{\mathcal{H}}$ that satisfies an assumption regarding the completeness of sample paths for random solutions. The conditions of Assumption 2 help in simplifying proofs related to viability and reachability probabilities and all the modified systems considered for the robustness analysis in the next section will satisfy the conditions of Assumption 2.

Assumption 2: The data of the SHS $\tilde{\mathcal{H}}$ are such that, for every random solution $\mathbf{x}$ generated by $\tilde{\mathcal{H}}$, the random solution $\mathbf{x}$ is almost surely complete.

The probability bounds in this section are generated for the system $\tilde{\mathcal{H}}$. We now present a series of bounds related to viability and reachability probabilities in this section and the proofs are presented later in the appendix. The results stated in this section are presented in [18] without proofs. In this paper, we provide detailed proofs for the results in [18] and illustrate the applications of the results in the context of robustness analysis and the development of converse Lyapunov theorems.

The first result establishes an equivalent characterization for the quantity $\widehat{m}_{\subset S}(x)$ defined in (4) for every closed set $S \subset \mathbb{R}^{n}$ and $x \in \mathbb{R}^{n}$.

Proposition 3. Let Assumption 2 hold, $S \subset \mathbb{R}^{n}$ be closed and $x \in \mathbb{R}^{n}$. Then, there exists a random solution $\mathbf{x}^{*} \in \mathcal{S}_{r}(x)$ such that

$$
\widehat{m}_{\subset S}(x)=\mathbb{P}\left(\operatorname{graph}\left(\mathbf{x}^{*}\right) \subset\left(\mathbb{R}^{2} \times S\right)\right)=\sup _{\mathbf{x} \in \mathcal{S}_{r}(x)} \mathbb{P}\left(\operatorname{graph}(\mathbf{x}) \subset\left(\mathbb{R}^{2} \times S\right)\right) .
$$

The following result when applied with the set $S=\mathbb{R}^{n} \backslash \mathcal{O}$ gives an alternative characterization of recurrence of the set $\mathcal{O}$ similar to [26, Lemma 3] and is a crucial intermediary step for proving the robustness results in Section 7.

Proposition 4. Let Assumption 2 hold and $S \subset \mathbb{R}^{n}$ be closed. If there exists $\gamma<1$ such that $\sup _{x \in S} \widehat{m}_{\subset S}(x) \leq$ $\gamma$, then $\widehat{m}_{\subset S}(x)=0$ for all $x \in S$.

The next result is motivated by the result in [23, Lemma 3] and is similar in nature to the semi-group property for non-stochastic systems.

Proposition 5. Let Assumption 2 hold. For closed sets $S_{0}, S_{1} \subset \mathbb{R}^{n}$ and $\left(k_{1}, k_{2}, x\right) \in \mathbb{Z}_{\geq 0} \times \mathbb{Z}_{\geq 0} \times \mathbb{R}^{n}$,

$$
m_{\subset S_{0}}\left(k_{1}+k_{2}, x\right) \leq m_{\subset S_{1}}\left(k_{1}, x\right)+\sup _{\xi \in \mathbb{R}^{n} \backslash S_{1}} m_{\subset S_{0}}\left(k_{2}, \xi\right)
$$


We now present a result that relates the viability and reachability probabilities. A similar result for discrete-time stochastic systems is in [23, Lemma 1].

Proposition 6. Let Assumption 2 hold. For closed sets $S, S_{1}, S_{2} \subset \mathbb{R}^{n}$ such that $S \subset S_{1} \cup S_{2}$ and for each $x \in \mathbb{R}^{n}$ and $\tau \geq 0$,

$$
m_{\subset S}(\tau, x) \leq m_{\subset S_{1}}(\tau, x)+m_{\cap S_{2}}(\tau, x)
$$

The next result establishes that the reachability probabilities $m_{\cap S}(\tau, x)$ can be made arbitrarily small for a fixed $\tau \geq 0$ and initial conditions $x$ in a compact set, when the set $S=\mathbb{R}^{n} \backslash R \mathbb{B}^{o}$ by choosing $R>0$ sufficiently large. The proof is omitted as it follows directly along the same lines as [23, Lemma 4] using the fact that the reachable set from a compact set of initial conditions for finite time is bounded for $x \in C, \dot{x} \in F(x)$ using [2, Lemma 6.16], the local boundedness $G$ and the dynamic programming methods in [2, Section 8.1].

Proposition 7. For each $k \in \mathbb{Z}_{\geq 0}, \varepsilon>0$ and $r>0$ there exists $R>0$ such that, with $S=\mathbb{R}^{n} \backslash R \mathbb{B}^{o}$, $m_{\cap S}(k, x) \leq \varepsilon$ for all $x \in r \mathbb{B}$.

\section{Robustness of recurrence}

In this section we establish robustness of the recurrence property to various state dependent perturbations. We prove robustness of the recurrence property to three different types of perturbations. Firstly, we establish that recurrence of an open bounded set implies recurrence of a smaller open set within the original set. This type of result can be viewed as robustness to perturbations in the set. Secondly, we prove recurrence is preserved when the data of the SHS is modified to slow down recurrence. Slowing down the recurrence property loosely means that we make quantities related to the average worst case first hitting time to the recurrent set for solutions from every initial condition increase with the distance of the initial condition to the recurrent set. Finally, we show that by perturbing the system data in a sufficiently small manner we preserve recurrence. This property establishes robustness of recurrence to measurement noise, additive disturbances and parametric uncertainty in the system data.

In this section, we will work with SHS for which the maximal random solutions have almost surely complete sample paths. The modification of the system $\mathcal{H}$ detailed below will preserve the recurrence property. If the open, bounded set $\mathcal{O}$ is globally recurrent for $\mathcal{H}$, consider the inflated system

$$
\widehat{\mathcal{H}}:=\left(C, F, \mathbb{R}^{n}, \widehat{G}, \mu\right)
$$

where $\widehat{G}(x, v)=G_{1}(x, v) \cup G_{2}(x)$ with $G_{1}(x, v)=G(x, v)$ for $x \in D, G_{1}(x, v)=\varnothing$ for $x \notin D$, and $G_{2}(x)=\left\{x^{*}\right\}$ for some $x^{*} \in \mathcal{O}$ and for all $x \in \mathbb{R}^{n}$. We note that the choice of $x^{*} \in \mathcal{O}$ determines the system data for $\widehat{\mathcal{H}}$. 
From the data of the hybrid system $\widehat{\mathcal{H}}$, recurrence of the set $\mathcal{O}$ for $\mathcal{H}$ and solutions of (1a) not exhibiting finite escape times it follows that for every random solution of $\widehat{\mathcal{H}}$ that is maximal, the sample paths are almost surely complete. The proof of the next result follows directly using [23, Prop 2] and 20, Prop 14.11 b].

Lemma 1. The data of the SHS $\widehat{\mathcal{H}}$ in $\sqrt{60}$ satisfies Standing Assumption 1 and Assumption 2.

The following result establishes that the recurrence property is preserved by the augment system $\widehat{\mathcal{H}}$ and the proof basically follows from the observation that maximal solutions of $\widehat{\mathcal{H}}$ contain solutions of $\mathcal{H}$ augmented with additional jumps to the recurrent set.

Lemma 2. If the open, bounded set $\mathcal{O}$ is globally recurrent for $\mathcal{H}$ in $(2)$, then $\mathcal{O}$ is globally recurrent for $\widehat{\mathcal{H}}$.

We can also observe that since the solutions of $\mathcal{H}$ are a subset of solutions of $\widehat{\mathcal{H}}$, if any set $\widehat{\mathcal{O}}$ is globally recurrent for $\widehat{\mathcal{H}}$, then $\widehat{\mathcal{O}}$ is also globally recurrent for $\mathcal{H}$.

Example 3: Consider the SHS $\mathcal{H}$ in Example 1. Now consider the inflated system $\widehat{\mathcal{H}}=\left(C, f, \mathbb{R}^{n}, \widehat{G}, \mu\right)$, where $\widehat{G}(x, v)=g(x, v) \cup G_{2}(x)$ for $x \in \mathbb{R}^{n}$ with $G_{2}(x)=\{0\}$. It follows that solutions generated by the augmented system $\widehat{\mathcal{H}}$ that are not solutions of the system $\mathcal{H}$ jump to the origin through the mapping $G_{2}$. Hence, global recurrence of the set $\mathcal{O}=(-\epsilon, \epsilon)$ where $0<\epsilon<1$ is preserved for the inflated system $\widehat{\mathcal{H}}$.

\subsection{Robustness to perturbations of the set}

The probabilities used in this subsection are generated using the system $\widehat{\mathcal{H}}$ for which the random solutions have almost surely complete sample paths. We define

$$
\tilde{m}_{\subset S}(\ell, \xi):=\sup _{\mathbf{x} \in \mathcal{S}_{r}(\xi)} \mathbb{P}\left(\operatorname{graph}(\mathbf{x}) \cap\left(\Gamma_{<\ell} \times \mathbb{R}^{n}\right) \subset\left(\mathbb{R}^{2} \times S\right)\right) .
$$

The motivation for defining the above quantity which is greater than or equal to $m_{\subset S}(\ell, \xi)$ is that we can apply the sequential compactness results developed in [27] to prove the next result. We establish that finite time viability probabilities related to a perturbation of a set $S$ from a compact set of initial conditions can be made arbitrarily close to probabilities related to the original set $S$ provided the perturbation is small enough.

Proposition 8. Let $S \subset \mathbb{R}^{n}$ be closed. For each $(\ell, \rho) \in \mathbb{Z}_{\geq 0} \times \mathbb{R}_{>0}$ and $K \subset \mathbb{R}^{n}$ compact there exists a $\varepsilon>0$ such that, for every $x \in K$,

$$
m_{\subset S+\varepsilon \mathbb{B}}(\ell, x) \leq \max _{\xi \in K} \tilde{m}_{\subset S}(\ell, \xi)+\rho .
$$

We now state the first main result related to robustness of the recurrence property. The following theorem establishes that recurrence of an open, bounded set implies the existence of a smaller recurrent set within the original set. The proof is presented in the appendix. 
Theorem 1. Let the open bounded set $\mathcal{O} \subset \mathbb{R}^{n}$ be globally recurrent for $\widehat{\mathcal{H}}$ in $(6)$. Then, there exists an open bounded set $\widehat{\mathcal{O}}$ and $\varepsilon>0$ such that $\widehat{\mathcal{O}}+\varepsilon \mathbb{B}^{o} \subset \mathcal{O}$ and $\widehat{\mathcal{O}}$ is globally recurrent for $\widehat{\mathcal{H}}$.

Example 4: Consider the SHS $\mathcal{H}$ in Example 1. It follows from Example 3 that the set $\mathcal{O}=(-\epsilon, \epsilon)$, where $0<\epsilon<1$ is globally recurrent for $\widehat{\mathcal{H}}$. It can be easily observed from the discrete-time dynamics that solutions exhibit jumps to the origin in an almost sure sense and hence the set $\widehat{\mathcal{O}}:=\{x:|x|<\epsilon / 2\}$ is also globally recurrent for $\widehat{\mathcal{H}}$ and satisfies $\widehat{\mathcal{O}}+(\epsilon / 3) \mathbb{B}^{\circ} \subset \mathcal{O}$.

\subsection{Robustness to slowing down recurrence}

The next inflation of the data of $\widehat{\mathcal{H}}$ results in preserving recurrence while making certain quantities related to the average value of worst case first hitting time for solutions to the set $\mathcal{O}$ grow unbounded in the distance of the state to the set $\mathcal{O}$. This result is important in the context of developing converse Lyapunov theorems with radially unbounded Lyapunov functions. Typically, for converse Lyapunov theorems involving the recurrence property the Lyapunov function candidate is related to the expected value of the first hitting time to the set $\mathcal{O}$. Consequently, for certain systems that allow for jumps to the recurrent set from arbitrarily large initial conditions, such a function will not be radially unbounded. The construction of the next inflation of the data of $\widehat{\mathcal{H}}$ is to prevent such behavior and ensure the Lyapunov function will be radially unbounded. See [18, 26] and [28] for more details.

For $\nu \in \mathcal{K}_{\infty}$, define the continuous set-valued mapping $M_{\nu}(x):=\left\{x^{*}\right\}+\nu\left(\left|x-x^{*}\right|\right) \mathbb{B}$ for $x^{*} \in \mathbb{R}^{n}$. Consider the inflated mapping

$$
\widehat{\mathcal{H}}_{\nu}:=\left(C, F, \mathbb{R}^{n}, \widehat{G}_{\nu}, \mu\right)
$$

where $\widehat{G}(x, v)=G_{1}(x, v) \cup M_{\nu}(x)$. The proof of the next result is very similar to Lemma 1

Lemma 3. For every $\nu \in \mathcal{K}_{\infty}$, the data of the $S H S \widehat{\mathcal{H}}_{\nu}$ in (8) satisfies Standing Assumption 1 and Assumption 2.

The next theorem claims the existence of a $\nu \in \mathcal{K}_{\infty}$ small enough to preserve recurrence of the set $\mathcal{O}$ for the inflated system $\widehat{\mathcal{H}}_{\nu}$ if $\mathcal{O}$ is globally recurrent for $\widehat{\mathcal{H}}$ and $x^{*} \in \mathcal{O}$. In essence, the result states that the recurrence property can be slowed in terms of average value of the worst case, first hitting time of the solutions to the set $\mathcal{O}$. By introducing the mapping $M_{\nu}$, for solutions starting from large initial conditions, the worst case time required to hit the set $\mathcal{O}$ is proportional to the distance of the initial conditions from the set $\mathcal{O}$. Hence, the average value of the worst case first hitting time increases with the distance to the set $\mathcal{O}$ and consequently slows down the recurrence property. A similar result is established for stochastic difference inclusions in [26, Theorem 4] and the proof presented in the appendix differs only in the construction of the function $\nu$.

Theorem 2. Let the open, bounded set $\mathcal{O} \subset \mathbb{R}^{n}$ be globally recurrent for $\widehat{\mathcal{H}}$. Then, for any $x^{*} \in \mathcal{O}$, there exists $\nu \in \mathcal{K}_{\infty}$ such that $\mathcal{O}$ is globally recurrent for $\widehat{\mathcal{H}}_{\nu}$ in (8). 


\subsection{Robustness to perturbations of system data}

Finally, we analyze the robustness of recurrence to sufficiently small state dependent perturbations. For a continuous, positive-valued function $\delta: \mathbb{R}^{n} \rightarrow \mathbb{R}_{>0}$, we denote the perturbed version of $\widehat{\mathcal{H}}$ by

$$
\widehat{\mathcal{H}}_{\delta}:=\left(C_{\delta}, F_{\delta}, \mathbb{R}^{n}, \widehat{G}_{\delta}, \mu\right)
$$

with the data defined as

$$
\begin{aligned}
C_{\delta} & :=\left\{x \in \mathbb{R}^{n}:(x+\delta(x) \mathbb{B}) \cap C \neq \varnothing\right\} \\
F_{\delta}(x) & :=\overline{\operatorname{con}} F((x+\delta(x) \mathbb{B}) \cap C)+\delta(x) \mathbb{B} \\
\widehat{G}_{\delta}(x, v) & :=\left\{w \in \mathbb{R}^{n}: w \in g+\delta(g) \mathbb{B}, g \in \widehat{G}(x+\delta(x) \mathbb{B}, v)\right\}
\end{aligned}
$$

where $\overline{\mathrm{con}}$ refers to the closed convex hull. The perturbations can be used to capture measurement errors and modeling uncertainties affecting the system data. The next result follows from [10, Proposition 6.28] and [23, Prop 8].

Lemma 4. For every continuous $\delta: \mathbb{R}^{n} \rightarrow \mathbb{R}_{>0}$, the data of the hybrid system $\widehat{\mathcal{H}}_{\delta}$ in $(9)$ satisfies Standing Assumption 1 and Assumption 2.

The next result establishes closeness of probabilities between the perturbed and unperturbed SHS. For constant perturbations we use $\delta(x) \equiv \delta$ for all $x \in \mathbb{R}^{n}$. In this subsection we denote the probabilities generated by the system $\widehat{\mathcal{H}}_{\delta}$ with the subscript $\delta$.

Proposition 9. Let $S \subset \mathbb{R}^{n}$ be closed. For each $(\ell, \rho) \in \mathbb{Z}_{\geq 0} \times \mathbb{R}_{>0}$ and $K \subset \mathbb{R}^{n}$ compact there exists a $\delta>0$ such that, for every $x \in K$,

$$
m_{\delta, \subset S}(\ell, x) \leq \sup _{\xi \in K} \tilde{m}_{\subset S}(\ell, \xi)+\rho .
$$

The next result establishes that recurrence of the open, bounded $\mathcal{O}$ set can be preserved when the state dependent perturbations are sufficiently small. The proof presented in the appendix follows along the same lines as [26, Thm 5].

Theorem 3. Let the open bounded set $\mathcal{O} \subset \mathbb{R}^{n}$ be globally recurrent for $\widehat{\mathcal{H}}$. Then, there exists a continuous function $\delta: \mathbb{R}^{n} \rightarrow \mathbb{R}_{>0}$ such that $\mathcal{O}$ is globally recurrent for $\widehat{\mathcal{H}}_{\delta}$ in $(9)$.

\section{Application to Converse theorems}

In this section, we briefly show the relationship between the robustness results in the previous section and the development of a converse Lyapunov theorem for recurrence. We first state a Lyapunov function based characterization of recurrence for open, bounded sets. A smooth function $V: \mathbb{R}^{n} \rightarrow \mathbb{R}_{\geq 0}$ is a Lyapunov 
function with respect to the set $\mathcal{O}$ for $\mathcal{H}$ if it is radially unbounded and there exists a continuous function $\rho: \mathbb{R}^{n} \rightarrow \mathbb{R}_{>0}$ and $\mu>0$ such that

$$
\begin{aligned}
\langle\nabla V(x), f\rangle & \leq-\rho(x)+\mu \mathbb{I}_{\mathcal{O}}(x), \forall x \in C, f \in F(x) \\
\int_{\mathbb{R}^{m}} \max _{g \in G(x, v)} V(g) \mu(d v) & \leq V(x)-\rho(x)+\mu \mathbb{I}_{\mathcal{O}}(x), \forall x \in D .
\end{aligned}
$$

It is established in [2] that the existence of $V$ satisfying the above conditions implies $\mathcal{O}$ is globally recurrent. We provide a brief overview of how the robustness results established in this paper will apply to the development of converse Lyapunov theorems. In [28, a complete presentation of the converse theorem is provided. We start from the assumption that $\mathcal{O}$ is recurrent for $\mathcal{H}$. The converse theorem will now establish the existence of a Lyapunov function satisfying the above decrease conditions.

Since $\mathcal{O}$ is recurrent for $\mathcal{H}$, it is recurrent for $\widehat{\mathcal{H}}$ by Lemma 2 . Then, Theorem 1 implies that there exists $\widehat{\mathcal{O}}$ and $\epsilon>0$ such that $\widehat{\mathcal{O}}+\epsilon \mathbb{B}^{o} \subset \mathcal{O}$ and $\widehat{\mathcal{O}}$ is recurrent for $\widehat{\mathcal{H}}$. Theorem 2 establishes that there exists $\nu \in \mathcal{K}_{\infty}$ such that $\widehat{\mathcal{O}}$ is recurrent for $\widehat{\mathcal{H}}_{\nu}$. Finally, Theorem 3 proves that there exists $\delta: \mathbb{R}^{n} \rightarrow \mathbb{R}_{>0}$ such that $\widehat{\mathcal{O}}$ is recurrent for $\widehat{\mathcal{H}}_{\nu, \delta}$. The goal is to construct a preliminary Lyapuov function candidate $V_{0}: \mathbb{R}^{n} \rightarrow \mathbb{R}_{\geq 0}$ (possibly non-smooth) which is radially unbounded and satisfies

$$
\begin{aligned}
D V_{0}(x) & \leq-\rho_{0}(x), \forall x \in C \backslash \widehat{\mathcal{O}}, \\
\int_{\mathbb{R}^{m}} \max _{g \in G(x, v) \backslash \widehat{\mathcal{O}}} V_{0}(g) \mu(d v) & \leq V_{0}(x)-\rho_{0}(x), \forall x \in D \backslash \widehat{\mathcal{O}},
\end{aligned}
$$

where $D$ refers to the Dini derivative and $\rho_{0}$ is a positive function characterizing decrease properties. Slowing down recurrence using Theorem 2 will help to guarantee that $V_{0}$ is radially unbounded, particularly when $V_{0}$ is related to the expected value of the worst first hitting time for solutions to the set $\widehat{\mathcal{O}}$. Then, we smooth the Lyapunov function $V_{0}$ that certifies $\widehat{\mathcal{O}}$ is recurrent for $\widehat{\mathcal{H}}_{\nu, \delta}$ to get a smooth function $V_{s}(x):=$ $\int_{\mathbb{R}^{n}} V_{0}(x+\sigma(x) \eta) \psi(\eta) d \eta$ for appropriately chosen functions $\sigma, \psi$. Then $V_{s}$ would be a smooth Lyapunov function with respect to $\mathcal{O}$ for the system $\mathcal{H}$. The structure of $V_{s}$ is motivated by [26] and [23].

Finally, we explain briefly the structure of the preliminary Lyapunov function $V_{0}$ that is considered in 28]. The function $V_{0}(x)=\int_{0}^{\infty} \kappa^{\prime}(\tau) m_{\subset \mathbb{R}^{n} \backslash \widehat{\mathcal{O}}}(\tau, x) d \tau+\alpha \mathbb{I}_{\mathbb{R}^{n} \backslash \widehat{\mathcal{O}}}(x)+\beta$ for some appropriately chosen $\alpha, \beta>0, \kappa \in \mathcal{K}_{\infty}$. We now explain the motivation for the structure of $V_{0}$. For recurrence of open, bounded sets in non-stochastic systems, it is established in [12] and [16. Thm 11.2.1] that the (worst-case) first hitting time for solutions to the recurrent set is a Lyapunov function candidate. A natural extension to the case of stochastic systems would be to consider the average value of the (worst-case) first hitting time for solutions to the recurrent set as a Lyapunov function candidate. In general, the average value of the (worst-case) first hitting time for solutions to the recurrent set need not be finite. In fact, it is finite and well defined only if a stronger property like positive recurrence of the set is assumed. The function $V_{0}$ is closely related to the average value of the average value of the (worst-case) first hitting time for solutions to the recurrent set and the role of the function $\kappa$ is to make the function $V_{0}$ well defined. 


\section{Conclusion}

We have presented results on robustness for a weak stochastic property called recurrence for a class of stochastic hybrid systems satisfying mild regularity properties. In particular, we have established that recurrence of an open bounded set implies recurrence of a smaller set contained in the original set, recurrence is robust to sufficiently small perturbations of the nominal system data that can slow down the recurrence property and recurrence is robust to sufficiently small modeling and measurement uncertainties. Future work will focus on applying the robustness results established in this paper in the context of developing converse Lyapunov theorems for the recurrence property as outlined in [18].

\section{Appendix}

\subsection{Proof of Proposition 2}

1) $\Rightarrow 2$ ) Since $\mathcal{O}$ is globally recurrent for $\mathcal{H}$, from Lemma $2, \mathcal{O}$ is globally recurrent for $\widehat{\mathcal{H}}$ for some $x^{*} \in \mathcal{O}$. Hence, for every $x \in \mathbb{R}^{n}$ and $\mathbf{x} \in \mathcal{S}_{r}(x)$ (generated by $\widehat{\mathcal{H}}$ ), we have

$$
\mathbb{P}\left(\operatorname{graph}(\mathbf{x}) \cap\left(\mathbb{R}^{2} \times \mathcal{O}\right)\right)=1 .
$$

Then, it follows that for every $x \in \mathbb{R}^{n}$ and $\mathbf{x} \in \mathcal{S}_{r}(x)$ (generated by $\widehat{\mathcal{H}}$ ), we have

$$
\mathbb{P}\left(\operatorname{graph}(\mathbf{x}) \subset\left(\mathbb{R}^{2} \times \mathbb{R}^{n} \backslash \mathcal{O}\right)\right)=0
$$

It follows from Proposition 3 that for every $x \in \mathbb{R}^{n}$, we have $\widehat{m}_{\subset \mathbb{R}^{n} \backslash \mathcal{O}}(x)=0$ for the SHS $\widehat{\mathcal{H}}$. Since the solutions

of $\mathcal{H}$ are also solutions of $\widehat{\mathcal{H}}$, it follows that $m_{\subset \mathbb{R}^{n} \backslash \mathcal{O}, \mathcal{H}}(\tau, x) \leq m_{\subset \mathbb{R}^{n} \backslash \mathcal{O}, \widehat{\mathcal{H}}}(\tau, x)$ for every $(\tau, x) \in \mathbb{R}_{\geq 0} \times \mathbb{R}^{n}$ and consequently for every $x \in \mathbb{R}^{n}, \widehat{m}_{\subset \mathbb{R}^{n} \backslash \mathcal{O}, \mathcal{H}}(x)=0$.

$2) \Rightarrow 3$ ) Follows from the proof of [23, Prop 5] using the upper semicontinuity of $(\tau, x) \mapsto m_{\subset \mathbb{R}^{n} \backslash \mathcal{O}}(\tau, x)$.

$3) \Rightarrow 1$ ) Follows from the definition of $m_{\subset \mathbb{R}^{n} \backslash \mathcal{O}}(\tau, x)$ in $[3]$, the definition of uniform global recurrence and Proposition 1.

\subsection{Proof of Proposition 3}

We recall the definition of $\tilde{m}$ in 7 related to the viability probabilities for a closed set $S$ :

$$
\tilde{m}_{\subset S}(\ell, \xi):=\sup _{\mathbf{x} \in \mathcal{S}_{r}(\xi)} \mathbb{P}\left(\operatorname{graph}(\mathbf{x}) \cap\left(\Gamma_{<\ell} \times \mathbb{R}^{n}\right) \subset\left(\mathbb{R}^{2} \times S\right)\right) .
$$

Next, we observe from the definition of $\widehat{m}$ in (4), and (3), (7) that for any closed set $S \subset \mathbb{R}^{n}$ and $x \in \mathbb{R}^{n}$,

$$
\widehat{m}_{\subset S}(x)=\lim _{\tau \rightarrow \infty} \tilde{m}_{\subset S}(\tau, x) .
$$

We now establish using sequential compactness results in [19] that the supremum in the characterization of $\tilde{m}$ is achieved for some random solution. We first observe that for any event $A \in \mathcal{F}, \mathbb{P}(A)=\mathbb{E}\left[\mathbb{I}_{A}\right]$, where $\mathbb{I}_{A}$ is the indicator function for the set $A$. Let $\ell \geq 0$. For hybrid $\operatorname{arcs} \phi$, define the function $\varphi$ such that $\varphi(\phi)=1$ 
if $\operatorname{graph}(\phi) \cap\left(\Gamma_{<\ell} \times \mathbb{R}^{n}\right) \subset\left(\mathbb{R}^{2} \times S\right)$ and $\varphi(\phi)$ is equal to 0 otherwise. Let $x \in \mathbb{R}^{n}$ and $\tilde{m}_{\subset S}(\ell, x)=\Delta \geq 0$. If $\Delta=0$, then every solution $\mathbf{x} \in \mathcal{S}_{r}(x)$ achieves the supremum. We now consider the case when $\Delta>0$. Let $\Delta_{i}<\Delta, i \in \mathbb{Z}_{\geq 0}$ be a sequence that converges to the value $\Delta$. Then, there exists a sequence of solution $\mathbf{x}_{i}$ such that $\mathbb{E}\left[\varphi\left(\mathbf{x}_{i}\right)\right] \geq \Delta_{i}$. Then, from [19, Thm 1], it follows that there exists a random solution $\mathbf{x}^{*}$ such that $\mathbb{E}\left[\varphi\left(\mathbf{x}^{*}\right)\right] \geq \Delta$. Since $\Delta$ is the supremum, it follows that $\mathbb{E}\left[\varphi\left(\mathbf{x}^{*}\right)\right]=\Delta$ which establishes the result.

Next, we show that for every $x \in \mathbb{R}^{n}$ and closed set $S \subset \mathbb{R}^{n}$,

$$
\lim _{i \rightarrow \infty} \sup _{\mathbf{x} \in \mathcal{S}_{r}(x)} \mathbb{P}\left(\operatorname{graph}(\mathbf{x}) \cap\left(\Gamma_{<i} \times \mathbb{R}^{n}\right) \subset\left(\mathbb{R}^{2} \times S\right)\right)=\sup _{\mathbf{x} \in \mathcal{S}_{r}(x)} \mathbb{P}\left(\operatorname{graph}(\mathbf{x}) \subset\left(\mathbb{R}^{2} \times S\right)\right) .
$$

We first observe from the continuity of probability that

$$
\begin{aligned}
\sup _{\mathbf{x} \in \mathcal{S}_{r}(x)} \mathbb{P}\left(\operatorname{graph}(\mathbf{x}) \subset\left(\mathbb{R}^{2} \times S\right)\right) & =\sup _{\mathbf{x} \in \mathcal{S}_{r}(x)} \lim _{i \rightarrow \infty} \mathbb{P}\left(\operatorname{graph}(\mathbf{x}) \cap\left(\Gamma_{<i} \times \mathbb{R}^{n}\right) \subset\left(\mathbb{R}^{2} \times S\right)\right) \\
& \leq \lim _{i \rightarrow \infty} \sup _{\mathbf{x} \in \mathcal{S}_{r}(x)} \mathbb{P}\left(\operatorname{graph}(\mathbf{x}) \cap\left(\Gamma_{<i} \times \mathbb{R}^{n}\right) \subset\left(\mathbb{R}^{2} \times S\right)\right) .
\end{aligned}
$$

For $x \in \mathbb{R}^{n}$ and $i \in \mathbb{Z}_{\geq 0}$, let $\Delta_{i}=\tilde{m}_{\subset S}(i, x)$ and $\Delta=\lim _{i \rightarrow \infty} \tilde{m}_{\subset S}(i, x)$. Then, the sequence $\Delta_{i}$ converges to $\Delta$. For hybrid $\operatorname{arcs} \phi$, define the function $\varphi_{i}$ such that $\varphi_{i}(\phi)=1$ if $\operatorname{graph}(\phi) \cap\left(\Gamma_{<i} \times \mathbb{R}^{n}\right) \subset\left(\mathbb{R}^{2} \times S\right)$ and $\varphi_{i}(\phi)$ is equal to 0 otherwise. The function $\varphi$ is defined such that $\varphi(\phi)=1$ if $\operatorname{graph}(\phi) \subset\left(\mathbb{R}^{2} \times S\right)$ and $\varphi(\phi)$ is equal to 0 otherwise. It follows from the above discussion that there exists a random solution $\mathbf{x}_{i} \in \mathcal{S}_{r}(x)$ such that $\mathbb{E}\left[\varphi_{i}\left(\mathbf{x}_{i}\right)\right]=\Delta_{i}$. Then, from [19, Thm 1] and the proof of [19, Thm 6], there exists a solution $\mathbf{x}$ such that $\mathbb{E}[\varphi(\mathbf{x})] \geq \Delta$. Hence it follows from the continuity of probability that

$$
\begin{aligned}
\lim _{i \rightarrow \infty} \sup _{\mathbf{x} \in \mathcal{S}_{r}(x)} \mathbb{P}\left(\operatorname{graph}(\mathbf{x}) \cap\left(\Gamma_{<i} \times \mathbb{R}^{n}\right) \subset\left(\mathbb{R}^{2} \times S\right)\right) & =\Delta \\
& \leq \mathbb{E}[\varphi(\mathbf{x})]=\mathbb{P}\left(\operatorname{graph}(\mathbf{x}) \subset\left(\mathbb{R}^{2} \times S\right)\right) \\
& \leq \sup _{\mathbf{x} \in \mathcal{S}_{r}(x)} \lim _{i \rightarrow \infty} \mathbb{P}\left(\operatorname{graph}(\mathbf{x}) \cap\left(\Gamma_{<i} \times \mathbb{R}^{n}\right) \subset\left(\mathbb{R}^{2} \times S\right)\right) .
\end{aligned}
$$

The bound 10 now follows from 11 and 12 . Hence, we have

$$
\widehat{m}_{\subset S}(x)=\lim _{i \rightarrow \infty} \sup _{\mathbf{x} \in \mathcal{S}_{r}(x)} \mathbb{P}\left(\operatorname{graph}(\mathbf{x}) \cap\left(\Gamma_{<i} \times \mathbb{R}^{n}\right) \subset\left(\mathbb{R}^{2} \times S\right)\right)=\sup _{\mathbf{x} \in \mathcal{S}_{r}(x)} \mathbb{P}\left(\operatorname{graph}(\mathbf{x}) \subset\left(\mathbb{R}^{2} \times S\right)\right) .
$$

The proof of existence of a random solution $\mathrm{x}^{*} \in \mathcal{S}_{r}(x)$ such that

$$
\mathbb{P}\left(\operatorname{graph}\left(\mathbf{x}^{*}\right) \subset\left(\mathbb{R}^{2} \times S\right)\right)=\sup _{\mathbf{x} \in \mathcal{S}_{r}(x)} \mathbb{P}\left(\operatorname{graph}(\mathbf{x}) \subset\left(\mathbb{R}^{2} \times S\right)\right)
$$

follows along the same lines as the proof for the random solution achieving the supremum in the definition of $\tilde{m}_{\subset S}(\ell, x)$ and is thus omitted.

\subsection{Proof of Proposition 4}

Let $x \in \mathbb{R}^{n}$ and $\mathbf{x} \in \mathcal{S}_{r}(x)$. Let $\Omega_{\infty}^{\mathbf{x}}:=\left\{\omega: \operatorname{graph}(\mathbf{x}(\omega)) \subset \mathbb{R}^{2} \times S\right\}$. Define

$$
\begin{aligned}
\mathbf{T}(\omega) & :=\sup \left\{t \in \pi_{1}\left(\operatorname{graph}(\mathbf{x}(\omega)) \cap\left(\Gamma_{\leq 1} \times \mathbb{R}^{n}\right)\right)\right\} \\
\mathbf{J}(\omega) & :=\sup \left\{j \in \pi_{2}\left(\operatorname{graph}(\mathbf{x}(\omega)) \cap\left(\Gamma_{\leq 1} \times \mathbb{R}^{n}\right)\right)\right\} .
\end{aligned}
$$


It follows from [2, Prop 2.1] that $\mathbf{T}, \mathbf{J}$ are $\mathcal{F}_{1}$ measurable. Define the process $\mathbf{y} \operatorname{such}$ that $\operatorname{graph}(\mathbf{y}(\omega))=$ $\operatorname{graph}(\mathbf{x}(\omega))-(\mathbf{T}(\omega), \mathbf{J}(\omega), 0)$. Without loss of generality the hybrid time domain of $\mathbf{x}(\omega)$ restricted to at most one jump is given by $\bigcup_{i=0}^{1}\left(\left[\mathbf{t}_{i}(\omega), \mathbf{t}_{i+1}(\omega)\right] \times\{i\}\right)$ for $\mathcal{F}_{i-1}$ measurable random variables $\mathbf{t}_{i}$ for $i=\{1,2\}$ and $\mathbf{t}_{0} \equiv 0$. See [2, Section 9.1] for more details. For hybrid arc $\phi$, the function $\varphi$ is defined such that $\varphi(\phi)=1$ if $\operatorname{graph}(\phi) \subset\left(\mathbb{R}^{2} \times S\right)$ and $\varphi(\phi)$ is equal to 0 otherwise. Hence, the behavior of the random solution $\mathbf{x}$ as time approaches infinity can be determined by the solution $\mathbf{x}$ up to a hybrid time of length 1 and the behavior of solution $\mathbf{y}$. We then have from [2, eqn (77)] and the definition of the function $\varphi$ that

$$
\left.\mathbb{P}\left(\Omega_{\infty}^{\mathbf{x}}\right)=\mathbb{E}\left[\max _{i \in\{0,1\}} \Pi_{j=0}^{i} \mathbb{I}_{\subset \mathbb{R}^{2} \times S}\left(\operatorname{graph}_{j, 1}(\mathbf{x})\right) \mathbb{I}_{\cap \mathbb{R}^{2} \times \mathbb{R}^{n}}\left(\operatorname{graph}_{i}(\mathbf{x})\right) \mathbb{I}_{\mathbb{R}_{\leq 0}}\left(1-\mathbf{t}_{i+1}-i\right)\right) \mathbb{E}\left[\varphi(\mathbf{y}) \mid \mathcal{F}_{1}\right]\right]
$$

where

$$
\begin{aligned}
\operatorname{graph}_{i}(\mathbf{x}) & :=\operatorname{graph}(\mathbf{x}) \cap\left(\mathbb{R} \times\{i\} \times \mathbb{R}^{n}\right) \\
\operatorname{graph}_{j, 1}(\mathbf{x}) & :=\operatorname{graph}_{j}(\mathbf{x}) \cap\left(\Gamma_{\leq 1} \times \mathbb{R}^{n}\right)
\end{aligned}
$$

We now establish that for every $k \in \mathbb{Z}_{\geq 0}$ and $x \in S$,

$$
\widehat{m}_{\subset S}(x) \leq \gamma m_{\subset S}(k, x)
$$

The bound holds for $k=0$ and every $x \in S$ since $\widehat{m}_{\subset S}(x) \leq \gamma$ and $m_{\subset S}(0, x)=1$ for every $x \in S$. We assume that the bound holds for some $k$ and every $x \in S$. Then, let $x \in S$ and $\mathbf{x} \in \mathcal{S}_{r}(x)$. Then, from $[2$, Prop 9.1, eqn(77)], definition of infinite time viability probabilities, Proposition 3, the induction bound and the definition of viability probabilities we have

$$
\begin{aligned}
\mathbb{P}\left(\Omega_{\infty}^{\mathbf{x}}\right) & =\mathbb{E}\left[\max _{i \in\{0,1\}} \Pi_{j=0}^{i} \mathbb{I}_{\subset \mathbb{R}^{2} \times S}\left(\operatorname{graph}_{j, 1}(\mathbf{x})\right) \mathbb{I}_{\cap \mathbb{R}^{2} \times \mathbb{R}^{n}}\left(\operatorname{graph}_{i}(\mathbf{x})\right) \mathbb{I}_{\mathbb{R}_{\leq 0}}\left(1-\mathbf{t}_{i+1}-i\right) \mathbb{E}\left[\varphi(\mathbf{y}) \mid \mathcal{F}_{1}\right]\right] \\
& \leq \mathbb{E}\left[\max _{i \in\{0,1\}} \Pi_{j=0}^{i} \mathbb{I}_{\subset \mathbb{R}^{2} \times S}\left(\operatorname{graph}_{j, 1}(\mathbf{x})\right) \mathbb{I}_{\cap \mathbb{R}^{2} \times \mathbb{R}^{n}}\left(\operatorname{graph}_{i}(\mathbf{x})\right) \mathbb{I}_{\mathbb{R}_{\leq 0}}\left(1-\mathbf{t}_{i+1}-i\right) \widehat{m}_{\subset S}(\mathbf{y}(0,0))\right] \\
& \left.\leq \gamma \mathbb{E} \max _{i \in\{0,1\}} \Pi_{j=0}^{i} \mathbb{I}_{\subset \mathbb{R}^{2} \times S}\left(\operatorname{graph}_{j, 1}(\mathbf{x})\right) \mathbb{I}_{\cap \mathbb{R}^{2} \times \mathbb{R}^{n}}\left(\operatorname{graph}_{i}(\mathbf{x})\right) \mathbb{I}_{\mathbb{R}_{\leq 0}}\left(1-\mathbf{t}_{i+1}-i\right) m_{\subset S}(k, \mathbf{y}(0,0))\right] \\
& \leq \gamma m_{\subset S}(k+1, x)
\end{aligned}
$$

Since this is true for any $x \in S$ and $\mathbf{x} \in \mathcal{S}_{r}(x)$, it follows from Proposition 3 that

$$
\widehat{m}_{\subset S}(x) \leq \gamma m_{\subset S}(k+1, x)
$$

The bound (14 holds by induction. Then as $k \rightarrow \infty$ we have

$$
\widehat{m}_{\subset S}(x) \leq \gamma \widehat{m}_{\subset S}(x)
$$

Since $\gamma<1$, it implies that $\sup _{x \in S} \widehat{m}_{\subset S}(x)=0$. 


\subsection{Proof of Proposition 5}

Let $x \in \mathbb{R}^{n}$ and $\mathbf{x} \in \mathcal{S}_{r}(x)$. For $x \in \mathbb{R}^{n} \backslash S_{1}$, the bound holds automatically due to the mapping $\tau \mapsto$ $m_{\subset S_{0}}(\tau, x)$ being non-increasing. Now, we consider the case when $x \in S_{1}$ and $\mathbf{x} \in \mathcal{S}_{r}(x)$. We first show that $\mathbb{P}\left(\operatorname{graph}(\mathbf{x}) \cap\left(\Gamma_{\leq k_{1}+k_{2}} \times \mathbb{R}^{n}\right) \subset \mathbb{R}^{2} \times S_{0}\right) \leq \mathbb{P}\left(\operatorname{graph}(\mathbf{x}) \cap\left(\Gamma_{\leq k_{1}} \times \mathbb{R}^{n}\right) \subset \mathbb{R}^{2} \times S_{1}\right)+\sup _{\xi \in \overline{\mathbb{R}^{n} \backslash S_{1}}} m_{\subset S_{0}}\left(k_{2}, \xi\right)$.

Let $\Omega_{0}:=\left\{\omega: \operatorname{graph}(\mathbf{x}) \cap\left(\Gamma_{\leq k_{1}+k_{2}} \times \mathbb{R}^{n}\right) \subset \mathbb{R}^{2} \times S_{0}\right\}, \Omega_{1}:=\left\{\omega: \operatorname{graph}(\mathbf{x}) \cap\left(\Gamma_{\leq k_{1}} \times \mathbb{R}^{n}\right) \subset \mathbb{R}^{2} \times S_{1}\right\}$ and $\Omega_{2}:=\left\{\omega: \omega \in \Omega_{0}, \operatorname{graph}(\mathbf{x}) \cap\left(\Gamma_{\leq k_{1}} \times \mathbb{R}^{n} \backslash S_{1}\right) \neq \varnothing\right\}$. We claim that $\Omega_{0} \subset\left(\Omega_{1} \cup \Omega_{2}\right)$. If not, there exists $\omega \in \Omega_{0}$ such that $\omega \notin \Omega_{1}$ and $\omega \notin \Omega_{2}$. Since $\omega \in \Omega_{0}$ and $\omega \notin \Omega_{1}$, then necessarily for some $(t, j)$, $\mathbf{x}_{\omega}(t, j) \in \mathbb{R}^{n} \backslash S_{1}$ and $t+j \leq k_{1}$ and $\mathbf{x}(\omega)$ remains in $S_{0}$ till hybrid time $k_{1}+k_{2}$. Hence, $\omega \in \Omega_{2}$. This leads to a contradiction and establishes the claim. Define

$$
\begin{aligned}
\mathbf{T}(\omega) & :=\inf \left\{t \in \pi_{1}\left(\operatorname{graph}(\mathbf{x}(\omega)) \cap\left(\Gamma_{\leq k_{1}} \times \mathbb{R}^{n} \backslash S_{1}\right)\right)\right\} \\
\mathbf{J}(\omega) & :=\inf \left\{j \in \pi_{2}\left(\operatorname{graph}(\mathbf{x}(\omega)) \cap\left(\Gamma_{\leq k_{1}} \times \mathbb{R}^{n} \backslash S_{1}\right)\right)\right\} .
\end{aligned}
$$

It follows from [2, Prop 2.1] that $\mathbf{T}, \mathbf{J}$ are $\mathcal{F}_{k_{1}}$ measurable random variables. Then, from the definition of the viability probabilities and with the convention that the probabilities are equal to zero starting from a null/ empty initial condition we have

$$
\begin{aligned}
\mathbb{P}\left(\Omega_{0}\right) & \leq \mathbb{P}\left(\Omega_{1} \cup \Omega_{2}\right) \leq \mathbb{P}\left(\Omega_{1}\right)+\mathbb{P}\left(\Omega_{2}\right) \\
& \leq \mathbb{P}\left(\Omega_{1}\right)+\mathbb{E}\left[m_{\subset S_{0}}\left(k_{2}, \mathbf{x}(\mathbf{T}, \mathbf{J})\right)\right] \\
& \leq \mathbb{P}\left(\Omega_{1}\right)+\sup _{\xi \in \overline{\mathbb{R}^{n} \backslash S_{1}}} m_{\subset S_{0}}\left(k_{2}, \xi\right) .
\end{aligned}
$$

Consequently, we have

$$
\mathbb{P}\left(\operatorname{graph}(\mathbf{x}) \subset \Gamma_{\leq k_{1}+k_{2}} \times S_{0}\right) \leq \mathbb{P}\left(\operatorname{graph}(\mathbf{x}) \subset \Gamma_{\leq k_{1}} \times S_{1}\right)+\sup _{\xi \in \overline{\mathbb{R}^{n} \backslash S_{1}}} m_{\subset S_{0}}\left(k_{2}, \xi\right) .
$$

The result of the proposition now follows as

$$
\begin{aligned}
m_{\subset S_{0}}\left(k_{1}+k_{2}, x\right) & =\sup _{\mathbf{x} \in \mathcal{S}_{r}(x)} \mathbb{P}\left(\operatorname{graph}(\mathbf{x}) \subset \Gamma_{\leq k_{1}+k_{2}} \times S_{0}\right) \\
& \leq \sup _{\mathbf{x} \in \mathcal{S}_{r}(x)} \mathbb{P}\left(\operatorname{graph}(\mathbf{x}) \subset \Gamma_{\leq k_{1}} \times S_{1}\right)+\sup _{\xi \in \mathbb{R}^{n} \backslash S_{1}} m_{\subset S_{0}}\left(k_{2}, \xi\right) \\
& =m_{\subset S_{1}}\left(k_{1}, x\right)+\sup _{\xi \in \mathbb{R}^{n} \backslash S_{1}} m_{\subset S_{0}}\left(k_{2}, \xi\right) .
\end{aligned}
$$

\subsection{Proof of Proposition 6}

The bound holds true for any $x \in \mathbb{R}^{n} \backslash S$ trivially. We now prove the result for $x \in S$. We claim that for every $x \in S$ and $\mathbf{x} \in \mathcal{S}_{r}(x)$,

$\mathbb{P}\left(\operatorname{graph}(\mathbf{x}) \cap\left(\Gamma_{\leq \tau} \times \mathbb{R}^{n}\right) \subset \mathbb{R}^{2} \times S\right) \leq \mathbb{P}\left(\operatorname{graph}(\mathbf{x}) \cap\left(\Gamma_{\leq \tau} \times \mathbb{R}^{n}\right) \subset \mathbb{R}^{2} \times S_{1}\right)+\mathbb{P}\left(\operatorname{graph}(\mathbf{x}) \cap\left(\Gamma_{\leq \tau} \times S_{2}\right) \neq \varnothing\right)$. 
Let $\Omega_{0}:=\left\{\omega: \operatorname{graph}(\mathbf{x}(\omega)) \cap\left(\Gamma_{\leq \tau} \times \mathbb{R}^{n}\right) \subset \mathbb{R}^{2} \times S\right\}, \Omega_{1}:=\left\{\omega: \operatorname{graph}(\mathbf{x}(\omega)) \cap\left(\Gamma_{\leq \tau} \times \mathbb{R}^{n}\right) \subset \mathbb{R}^{2} \times S_{1}\right\}$ $\Omega_{2}:=\left\{\omega: \operatorname{graph}(\mathbf{x}(\omega)) \cap\left(\Gamma_{\leq \tau} \times S_{2}\right) \neq \varnothing\right\}$. We claim that $\Omega_{0} \subset\left(\Omega_{1} \cup \Omega_{2}\right)$. If not, for some $\omega \in \Omega_{0}$ we have $\omega \notin \Omega_{1}$ and $\omega \notin \Omega_{2}$. If $\omega \notin \Omega_{1}$, it implies that $\operatorname{graph}(\mathbf{x}(\omega)) \cap\left(\Gamma_{\leq \tau} \times\left(S \backslash S_{1}\right)\right) \neq \varnothing$. Since $S \subset S_{1} \cup S_{2}$, this means that $\operatorname{graph}(\mathbf{x}(\omega)) \cap\left(\Gamma_{\leq \tau} \times S_{2}\right) \neq \varnothing$ and hence $\omega \in \Omega_{2}$. This leads to a contradiction. Then, $\mathbb{P}\left(\Omega_{0}\right) \leq \mathbb{P}\left(\Omega_{1}\right)+\mathbb{P}\left(\Omega_{2}\right)$. The result of the proposition then follows from taking the supremum over all possible random solutions from $x$ on both sides.

\subsection{Proof of Proposition 8}

We first claim that for each $(\ell, \rho) \in \mathbb{Z}_{\geq 0} \times \mathbb{R}_{>0}$ and $K \subset \mathbb{R}^{n}$ compact there exists a $\varepsilon>0$ such that, for every $x \in K$ compact and $\mathbf{x} \in \mathcal{S}_{r}(x)$,

$$
\mathbb{P}\left(\operatorname{graph}(\mathbf{x}) \cap\left(\Gamma_{<\ell} \times \mathbb{R}^{n}\right) \subset \mathbb{R}^{2} \times(S+\varepsilon \mathbb{B})\right) \leq \max _{\xi \in K} \tilde{m}_{\subset S}(\ell, \xi)+\rho .
$$

If the claim is not true, then there exists $(\ell, \rho)$ and a compact set $K$ such that for every $i \in \mathbb{Z}_{\geq 1}$ we have for some $x_{i} \in K$ and $\mathbf{x}_{i} \in \mathcal{S}_{r}\left(x_{i}\right)$

$$
\mathbb{P}\left(\operatorname{graph}\left(\mathbf{x}_{i}\right) \cap\left(\Gamma_{<\ell} \times \mathbb{R}^{n}\right) \subset \mathbb{R}^{2} \times(S+1 / i \mathbb{B})\right)>\max _{\xi \in K} \tilde{m}_{\subset S}(\ell, \xi)+\rho .
$$

For hybrid $\operatorname{arcs} \phi$, define the function $\varphi_{i}$ such that $\varphi_{i}(\phi)=1$ if $\operatorname{graph}(\phi) \cap\left(\Gamma_{<\ell} \times \mathbb{R}^{n}\right) \subset \mathbb{R}^{2} \times(S+(1 / i) \mathbb{B})$ and 0 otherwise. Similarly, the function $\varphi$ is defined using set $S$. Then,

$$
\mathbb{E}\left[\varphi_{i}\left(\mathbf{x}_{i}\right)\right]>\max _{\xi \in K} \tilde{m}_{\subset S}(\ell, \xi)+\rho .
$$

From [19, Thm 1] and the proof of [19, Thm 6], we can establish that there exists a random solution $\mathbf{x}$ from $K$ such that

$$
\mathbb{E}[\varphi(\mathbf{x})] \geq \max _{\xi \in K} \tilde{m}_{\subset S}(\ell, \xi)+\rho .
$$

Since $\mathbb{E}[\varphi(\mathbf{x})] \leq \max _{\xi \in K} \tilde{m}_{\subset S}(\ell, \xi)$ and $\rho>0$, it leads to a contradiction that establishes the result. Then, we observe that

$$
\mathbb{P}\left(\operatorname{graph}(\mathbf{x}) \cap\left(\Gamma_{\leq \ell} \times \mathbb{R}^{n}\right) \subset \mathbb{R}^{2} \times(S+\varepsilon \mathbb{B})\right) \leq \mathbb{P}\left(\operatorname{graph}(\mathbf{x}) \cap\left(\Gamma_{<\ell} \times \mathbb{R}^{n}\right) \subset \mathbb{R}^{2} \times(S+\varepsilon \mathbb{B})\right) .
$$

The result now follows from the bound (15) by taking the supremum over all possible random solutions from the initial condition $x$ for the SHS $\widehat{\mathcal{H}}$.

\subsection{Proof of Lemma 1}

We first establish that Standing Assumption 1 is satisfied for $\widehat{\mathcal{H}}$. As the continuous-time dynamics of the system $\widehat{\mathcal{H}}$ is unchanged from $\mathcal{H}$ and the set $D=\mathbb{R}^{n}$ only condition 3 of Standing Assumption 1 needs to be verified. Since the graph of the union of two set-valued mappings is equal to the union of their graphs, it follows that $x \mapsto \widehat{G}(x, v)$ is outer semicontinuous. Finally, it follows from [15, Proposition 14.11(a)] that $v \mapsto \operatorname{graph}(\widehat{G}(, v))$ is measurable. Assumption 2 is satisfied trivially as for random solution $\mathbf{x}$, almost all sample paths of the random solution can be extended to be complete by augmenting additional jumps to $x^{*}$. 


\subsection{Proof of Lemma 2}

Since the flow map for the hybrid system $\widehat{\mathcal{H}}$ is the same as $\mathcal{H}$, the solutions generated by $\widehat{\mathcal{H}}$ do not exhibit finite escape times. We will now establish global recurrence of $\mathcal{O}$ for $\widehat{\mathcal{H}}$ by contradiction. If $\mathcal{O}$ is not globally recurrent for $\widehat{\mathcal{H}}$, then there exists $\rho>0$ and a random solution $\mathbf{x}$ such that $\mathbb{P}\left(\operatorname{graph}(\mathbf{x}) \subset \mathbb{R}^{2} \times\left(\mathbb{R}^{n} \backslash \mathcal{O}\right)\right) \geq \rho$. Without loss of generality we can assume that the solution $\mathbf{x}$ is maximal and almost surely the sample paths are complete. We also observe that for $\omega \in \Omega$ such that $\operatorname{graph}(\mathbf{x}(\omega)) \subset \mathbb{R}^{2} \times\left(\mathbb{R}^{n} \backslash \mathcal{O}\right), \mathbf{x}_{\omega}(t, j) \neq x^{*}$ for all $(t, j) \in \operatorname{dom}(\mathbf{x}(\omega))$. We now define a solution $\tilde{\mathbf{x}}$ for the system $\mathcal{H}$ using $\mathbf{x}$. For the case when $\omega$ is such that $\mathbf{x}_{\omega}(t, j+1)=G_{2}\left(\mathbf{x}_{\omega}(t, j)\right)=x^{*}$ occurs for the first time $(t, j) \in \operatorname{dom}(\mathbf{x}(\omega))$, we let $\tilde{\mathbf{x}}_{\omega}(\bar{t}, \bar{j})=\mathbf{x}_{\omega}(\bar{t}, \bar{j})$ for $\bar{t} \leq t, \bar{j} \leq j$ and the sample paths are stopped afterwards. Otherwise, we let $\tilde{\mathbf{x}}(\omega)=\mathbf{x}(\omega)$. It can be easily observed that $\tilde{\mathbf{x}}$ is a truncation of the solution $\mathbf{x}$, truncated at first jump times where the mapping $G_{2}$ is used in the sample paths. Then, we can establish that $\tilde{\mathbf{x}}$ satisfies $\mathcal{F}_{i}$ measurability of the mapping $\omega \mapsto \operatorname{graph}_{\leq i}(\tilde{\mathbf{x}}(\omega))$ from [2, Prop 2.1]. Let $\Omega_{i}:=\left\{\omega: \operatorname{graph}(\tilde{\mathbf{x}}(\omega)) \cap\left(\Gamma_{\geq i} \times \mathbb{R}^{n}\right) \neq \varnothing\right\}$ for $i \in \mathbb{Z}_{\geq 0}$. Then, $\Omega_{i} \in \mathcal{F}$ from [20, Thm 14.3(a), Prop 14.11(a)]. Since $\Omega_{i} \in \mathcal{F}$ for all $i \in \mathbb{Z}_{\geq 0}$, it follows that $\cap_{i} \Omega_{i} \in \mathcal{F}$. Let $\Omega_{2}:=\left\{\omega: \operatorname{graph}(\tilde{\mathbf{x}}(\omega)) \subset \mathbb{R}^{2} \times\left(\mathbb{R}^{n} \backslash \mathcal{O}\right)\right\}$. Then, $\Omega_{2} \in \mathcal{F}$ follows from [20, Thm 14.3(i)]. Then, necessarily we have $\mathbb{P}\left(\Omega_{c}\right) \geq \rho$ where $\Omega_{c}=\left\{\omega: \tilde{\mathbf{x}}(\omega)\right.$ is complete and $\left.\operatorname{graph}(\tilde{\mathbf{x}}(\omega)) \subset \mathbb{R}^{2} \times\left(\mathbb{R}^{n} \backslash \mathcal{O}\right)\right\}=\left(\cap_{i} \Omega_{i}\right) \cap \Omega_{2}$ and $\Omega_{c} \in \mathcal{F}$. This contradicts global recurrence of $\mathcal{O}$ for $\mathcal{H}$ and establishes the result.

\subsection{Proof of Theorem 1}

Let $\ell_{0} \in \mathbb{Z}_{>0}$ be such that $\tilde{m}_{\subset \mathbb{R}^{n} \backslash \mathcal{O}}\left(\ell_{0}, x\right) \leq 0.25 / 2$ for all $x \in \overline{\mathcal{O}+\mathbb{B}^{\circ}}$. This bound follows from the uniform global recurrence of the set $\mathcal{O}$. We now use the result of Proposition 8 with $K:=\overline{\mathcal{O}+\mathbb{B}^{\circ}}$ and $S=\mathbb{R}^{n} \backslash \mathcal{O}$. Then, there exists $\tilde{\varepsilon} \in(0,1)$ such that, for every $x \in K$,

$$
m_{\subset\left(\mathbb{R}^{n} \backslash \mathcal{O}\right)+\tilde{\varepsilon} \mathbb{B}}\left(\ell_{0}, x\right) \leq \max _{\xi \in \overline{\mathcal{O}+\mathbb{B}^{o}}} \tilde{m}_{\subset \mathbb{R}^{n} \backslash \mathcal{O}}\left(\ell_{0}, \xi\right)+0.25 / 2 .
$$

Define the open, bounded set $\widehat{\mathcal{O}}:=\mathbb{R}^{n} \backslash\left(\left(\mathbb{R}^{n} \backslash \mathcal{O}\right)+\tilde{\varepsilon} \mathbb{B}\right)$. Hence, it follows that for $\varepsilon=\tilde{\varepsilon} / 2>0, \widehat{\mathcal{O}}+\varepsilon \mathbb{B} \subset \mathcal{O}$. Since $\tilde{\varepsilon}<1, \mathcal{O} \subset \widehat{\mathcal{O}}+\mathbb{B}^{o}$. Then, for all $x \in \overline{\widehat{\mathcal{O}}+\mathbb{B}^{o}}$,

$$
m_{\subset \mathbb{R}^{n} \backslash \widehat{\mathcal{O}}}\left(\ell_{0}, x\right) \leq m_{\subset\left(\mathbb{R}^{n} \backslash \mathcal{O}\right)+\tilde{\varepsilon} \mathbb{B}}\left(\ell_{0}, x\right) \leq 0.25 .
$$

We now complete the proof as follows. Let $S_{i}:=\mathbb{R}^{n} \backslash\left(\widehat{\mathcal{O}}+i \mathbb{B}^{o}\right)$ be a sequence of closed sets for $i \in \mathbb{Z}_{\geq 0}$. Since $\mathcal{O} \subset \widehat{\mathcal{O}}+\mathbb{B}^{o}$, it follows from uniform global recurrence of the set $\mathcal{O}$ that for all $\xi \in \overline{\mathbb{R}^{n} \backslash S_{i+1}}$ there exists $\ell_{i} \in \mathbb{Z}_{>0}$ such that $m_{\subset S_{1}}\left(\ell_{i}, \xi\right) \leq 0.25$. Then, for all $i \in \mathbb{Z}_{\geq 1}, x \in \overline{\mathbb{R}^{n} \backslash S_{i+1}}$, we have from Proposition 5

$$
\begin{aligned}
m_{\subset S_{0}}\left(\ell_{i}+\ell_{0}, x\right) & \leq m_{\subset S_{1}}\left(\ell_{i}, x\right)+\sup _{\xi \in \mathbb{R}^{n} \backslash S_{1}} m_{\subset S_{0}}\left(\ell_{0}, \xi\right) \\
& \leq 0.5 .
\end{aligned}
$$

Then, from the monotonicity of the viability probabilities we have that for every $x \in \mathbb{R}^{n}, \widehat{m}_{\subset \mathbb{R}^{n} \backslash \widehat{\mathcal{O}}}(x) \leq 0.5$. Hence, it follows from Proposition 4 that for all $x \in \mathbb{R}^{n}, \widehat{m}_{\subset \mathbb{R}^{n} \backslash \widehat{\mathcal{O}}}(x)=0$. This equality implies that the set $\widehat{\mathcal{O}}$ is globally recurrent for $\widehat{\mathcal{H}}$ from Proposition 2 . 


\subsection{Proof of Theorem 2}

We denote the probabilities generated by the system $\widehat{\mathcal{H}}_{\nu}$ with the subscript $\nu$. Let $\mathcal{O}$ be the recurrent set. Now let $S_{i}:=\mathbb{R}^{n} \backslash\left(\mathcal{O}+i \mathbb{B}^{o}\right)$ be a sequence of closed sets and $0<\varepsilon_{i} \leq\left(\frac{1}{2}\right)^{i+2}$ for all $i \in \mathbb{Z}_{\geq 0}$. Then, for every $i \in \mathbb{Z}_{\geq 0}$, choose $\ell_{i}$ such that

$$
m_{\subset S_{i}}\left(\ell_{i}, x\right) \leq \frac{1}{2} \varepsilon_{i} \forall x \in \overline{\mathbb{R}^{n} \backslash S_{i+1}} .
$$

This bound follows from the uniform global recurrence of the set $\mathcal{O}$ for the system $\widehat{\mathcal{H}}$. Let $\beta_{i} \in \mathbb{Z}_{\geq 0}$. Then, choose $\beta_{i} \geq i+1$ such that, with $\nu(s)=s$ for all $s \geq 0$,

$$
m_{\nu, \cap S_{\beta_{i}}}\left(\ell_{i}, x\right) \leq \frac{1}{2} \varepsilon_{i} \quad \forall x \in \overline{\mathbb{R}^{n} \backslash S_{i+1}} .
$$

The values $\beta_{i}$ exists according to Proposition 7. Without loss of generality we can assume the function $i \mapsto \beta_{i}$ is strictly increasing and unbounded. Define $K_{i}:=\left(\overline{\mathcal{O}+\beta_{i} \mathbb{B}^{o}}\right) \backslash\left(\mathcal{O}+i \mathbb{B}^{o}\right)$.

We use the fact that under the conditions of Standing Assumption 1, the infinite time reachable set 1 ([10, Sec 6.3.2]) from $\overline{\mathcal{O}}$ denoted by $\Gamma$ is bounded for solutions of $\dot{x} \in F(x), x \in C$ (from [12, Prop. 2]). Uniform global recurrence of $\mathcal{O}$ implies that there exists a time $J_{i}>0$ such that each solution of $\dot{x} \in F(x), x \in C$ from $\overline{\mathcal{O}+\beta_{i} \mathbb{B}^{\circ}}$ reaches the set $\mathcal{O}$, or stops, within time $J_{i}$. Then, the reachable set in infinite time for $\dot{x} \in F(x), x \in C$ from $\overline{\mathcal{O}+\beta_{i} \mathbb{B}^{o}}$, is given by $\mathcal{R}\left(\overline{\mathcal{O}+\beta_{i} \mathbb{B}^{o}}\right)=\mathcal{R}_{\leq J_{i}}\left(\overline{\mathcal{O}+\beta_{i} \mathbb{B}^{o}}\right) \cup \Gamma$ where $\mathcal{R}_{\leq J_{i}}\left(\overline{\mathcal{O}+\beta_{i} \mathbb{B}^{o}}\right)$ is the reachable set within time $J_{i}$. It follows from [10, Lemma 6.16] that $\mathcal{R}\left(\overline{\widehat{\mathcal{O}}+\beta_{i} \mathbb{B}^{\circ}}\right)$ is bounded.

We define $\gamma_{i}:=\sup _{x \in \mathcal{R}\left(\overline{\mathcal{O}+\beta_{i} \mathbb{B}^{\circ}}\right)}\left|x-x^{*}\right|$ and $r_{i}:=\inf _{y \in \partial\left(\overline{\mathcal{O}+i \mathbb{B}^{\circ}}\right)}\left|y-x^{*}\right|$. Let $\nu \in \mathcal{K}_{\infty}$ with $\nu(s)<s$ for all $s>0$ and satisfy $\nu\left(\gamma_{i}\right)<r_{i} / 2$ for all $i \in \mathbb{Z}_{\geq 0}$.

Next we claim that for all $x \in \overline{\mathbb{R}^{n} \backslash S_{\beta_{i}}}$ and all $\tau \in \mathbb{Z}_{\geq 0}$,

$$
m_{\nu, \subset K_{i}}(\tau, x)=m_{\subset K_{i}}(\tau, x) .
$$

The proof of the above result follows exactly along the same line as [26, Thm 4] using induction and dynamic programming from [2, Sec 9] and is thus omitted.

Now using Proposition 6 , we have that for all $i \in \mathbb{Z}_{\geq 0}$ and every $x \in \overline{\mathbb{R}^{n} \backslash S_{i+1}}$,

$$
\begin{aligned}
m_{\nu, \subset S_{i}}\left(\ell_{i}, x\right) & \leq m_{\nu, \cap S_{\beta_{i}}}\left(\ell_{i}, x\right)+m_{\nu, \subset K_{i}}\left(\ell_{i}, x\right) \\
& \leq \frac{1}{2} \varepsilon_{i}+m_{\subset K_{i}}\left(\ell_{i}, x\right) \leq \frac{1}{2} \varepsilon_{i}+m_{\subset S_{i}}\left(\ell_{i}, x\right) \\
& \leq \varepsilon_{i} .
\end{aligned}
$$

Given $x \in \mathbb{R}^{n}$, let $i \in \mathbb{Z}_{\geq 1}$ be such that $x \in \overline{\mathbb{R}^{n} \backslash S_{i+1}}$. Then, we have $m_{\nu, \subset S_{i}}\left(\ell_{i}, x\right) \leq \varepsilon_{i}$, and similarly we have that for every $k \in\{0, . ., i-1\}, \sup _{\xi \in \overline{\mathbb{R}^{n} \backslash S_{k+1}}} m_{\nu, \subset S_{k}}\left(\ell_{k}, \xi\right) \leq \varepsilon_{k}$. Then, from Proposition 5 it follows

\footnotetext{
${ }^{1}$ The reachable set from a set $S$ within time $\tau$ is defined as $\mathcal{R}_{\leq \tau}(S):=\{\phi(t): \phi(0) \in S$ and $t \leq \tau\}$. The reachable set from a set $S$ in infinite hybrid time is $\cup_{\tau \geq 0} \mathcal{R}_{\tau}(S)$.
} 
that

$$
\begin{aligned}
m_{\nu, \subset S_{0}}\left(\sum_{j=0}^{i} \ell_{j}, x\right) & \leq m_{\nu, \subset S_{1}}\left(\sum_{j=1}^{i} \ell_{j}, x\right)+\sup _{\xi \in \mathbb{\mathbb { R }}^{n} \backslash S_{1}} m_{\nu, \subset S_{0}}\left(\ell_{0}, \xi\right) \\
& \leq m_{\nu, \subset S_{i}}\left(\ell_{i}, x\right)+\sum_{k=0}^{i-1} \sup _{\xi \in \mathbb{R}^{n} \backslash S_{k+1}} m_{\nu, \subset S_{k}}\left(\ell_{k}, \xi\right) \\
& \leq \sum_{k=0}^{i} \varepsilon_{k} \leq 0.5 .
\end{aligned}
$$

Hence, from the monotonicity of the viability probabilities we can conclude that $\widehat{m}_{\nu, \subset S_{0}}(x) \leq 0.5$ for all $x \in \mathbb{R}^{n}$. Then, from Proposition 4 it follows that $\widehat{m}_{\nu, \subset \mathbb{R}^{n} \backslash \mathcal{O}}(x)=0$ for every $x \in \mathbb{R}^{n}$. Global recurrence of $\mathcal{O}$ for $\mathcal{H}_{\nu}$ follows from Proposition 2 .

\subsection{Proof of Proposition 9}

We first claim that for each $(\ell, \rho) \in \mathbb{Z}_{\geq 0} \times \mathbb{R}_{>0}$ and $K \subset \mathbb{R}^{n}$ compact there exists a $\delta>0$ such that, for every $x \in K$ compact and $\mathbf{x} \in \mathcal{S}_{r}^{\delta}(x)$,

$$
\mathbb{P}\left(\operatorname{graph}(\mathbf{x}) \cap\left(\Gamma_{<\ell} \times \mathbb{R}^{n}\right) \subset \mathbb{R}^{2} \times S\right) \leq \sup _{\xi \in K} \tilde{m}_{\subset S}(\ell, \xi)+\rho
$$

where $\mathcal{S}_{r}^{\delta}(x)$ refers to the set of random solutions generated by the $\widehat{\mathcal{H}}_{\delta}$ system. If the claim is not true, then there exists $(\ell, \rho)$ and compact set $K$ such that for every $i \in \mathbb{Z}_{\geq 1}$ we have for some $x_{i} \in K$ and $\mathbf{x}_{i} \in \mathcal{S}_{r}^{(1 / i)}\left(x_{i}\right)$

$$
\mathbb{P}\left(\operatorname{graph}\left(\mathbf{x}_{i}\right) \cap\left(\Gamma_{<\ell} \times \mathbb{R}^{n}\right) \subset \mathbb{R}^{2} \times S\right)>\sup _{\xi \in K} \tilde{m}_{\subset S}(\ell, \xi)+\rho .
$$

Define the function $\varphi_{i}$ for hybrid $\operatorname{arc} \phi$ such that $\varphi(\phi)=1$ if $\operatorname{graph}(\phi) \cap\left(\Gamma_{<\ell} \times \mathbb{R}^{n}\right) \subset \mathbb{R}^{2} \times S$ and 0 otherwise. Then,

$$
\mathbb{E}\left[\varphi\left(\mathbf{x}_{i}\right)\right] \geq \sup _{\xi \in K} \tilde{m}_{\subset S}(\ell, \xi)+\rho .
$$

It follows from a simple extension of [19, Thm 1] to a class of perturbed systems (by augmenting the state variable with an additional perturbation variable similar to [19] [Section IV.B]) and the proof of [19, Thm 6] that there exists a random solution $\mathbf{x}$ from $K$ for the nominal system such that

$$
\mathbb{E}[\varphi(\mathbf{x})] \geq \sup _{\xi \in K} \tilde{m}_{\subset S}(\ell, \xi)+\rho .
$$

Since $\mathbb{E}[\varphi(\mathbf{x})] \leq \sup _{\xi \in K} \tilde{m}_{\subset S}(\ell, \xi)$ and $\rho>0$, it leads to a contradiction that establishes the claim. The result now follows from the bound (18) by taking the supremum over all possible random solutions from the initial condition $x$ for system $\widehat{\mathcal{H}}_{\delta}$.

\subsection{Proof of Theorem 3}

For $i \in \mathbb{Z}_{\geq 0}$, let $0<\varepsilon_{i} \leq(1 / 2)^{i+2}$ and $S_{i}:=\mathbb{R}^{n} \backslash\left(\mathcal{O}+i \mathbb{B}^{o}\right)$ be a sequence of closed sets. It follows from uniform global recurrence of $\mathcal{O}$ for $\widehat{\mathcal{H}}$ that there exists $\ell_{i}$ be such that $\sup _{\xi \in \overline{\mathbb{R}^{n} \backslash S_{i+1}}} \tilde{m}_{\subset S_{i}}\left(\ell_{i}, \xi\right) \leq \varepsilon_{i} / 2$. Then, let $\delta_{i}>0$ come from the application of Proposition 9 with the compact set $K=\overline{\mathbb{R}^{n} \backslash S_{i+1}}$ and $\rho=\varepsilon_{i} / 2$. 
Given $x \in \mathbb{R}^{n}$, define $i(x):=\min \left\{j \in \mathbb{Z}_{\geq 1}: x \in \overline{\mathbb{R}^{n} \backslash S_{j+1}}\right\}$. Then, we have $m_{\delta_{i(x)}, \subset S_{i(x)}}\left(\ell_{i(x)}, x\right) \leq \varepsilon_{i}$. Similarly we have that for every $k \in\{0, \ldots, i(x)-1\}$ there exists $\delta_{k}, \ell_{k}>0$ such that $\sup _{\xi \in \overline{\mathbb{R}^{n}} \backslash S_{k+1}} m_{\delta_{k}, \subset S_{k}}\left(\ell_{k}, \xi\right) \leq$ $\varepsilon_{k}$. Then, define a continuous state dependent perturbation $\delta: \mathbb{R}^{n} \rightarrow \mathbb{R}_{>0}$ as follows,

$$
\widehat{\delta}(x):=\min _{k \in\{0,1, \ldots, i(x)\}} \delta_{k}, \quad \delta(x):=\inf _{\xi \in \mathbb{R}^{n}}(\widehat{\delta}(\xi)+|\xi-x|) .
$$

It can be verified easily that the function $\delta$ satisfies the Lipschitz property globally with Lipschitz constant 1. Hence continuity of $\delta$ follows. Then, similar to the proof of Theorem 2 it follows from Proposition 5 that

$$
\begin{aligned}
m_{\delta, \subset S_{0}}\left(\sum_{j=0}^{i(x)} \ell_{j}, x\right) & \leq m_{\delta, \subset S_{1}}\left(\sum_{j=1}^{i(x)} \ell_{j}, x\right)+\sup _{\xi \in \mathbb{R}^{n} \backslash S_{1}} m_{\delta, \subset S_{0}}\left(\ell_{0}, \xi\right) \\
& \leq m_{\delta, \subset S_{i(x)}}\left(\ell_{i(x)}, x\right)+\sum_{k=0}^{i(x)-1} \sup _{\xi \in \mathbb{R}^{n} \backslash S_{k+1}} m_{\delta, \subset S_{k}}\left(\ell_{k}, \xi\right) \\
& \leq \sum_{k=0}^{i(x)} \varepsilon_{k} \leq 0.5 .
\end{aligned}
$$

Hence, from monotonicity of the viability probabilities we can conclude that $\widehat{m}_{\delta, \subset S_{0}}(x) \leq 0.5$ for all $x \in \mathbb{R}^{n}$. Then, it follows from Proposition 4 that for all $x \in \mathbb{R}^{n}, \widehat{m}_{\delta, \subset \mathbb{R}^{n} \backslash \mathcal{O}}(x)=0$. The result now follows from Proposition 2

\section{References}

[1] G. G. Yin, C. Zhu, Hybrid switching diffusions: properties and applications, Vol. 63, Springer, 2009.

[2] A. R. Teel, Lyapunov conditions certifying stability and recurrence for a class of stochastic hybrid systems, Annual Reviews in Control 37 (1) (2013) 1 - 24.

[3] M. H. Davis, Piecewise-deterministic Markov processes: A general class of non-diffusion stochastic models, Journal of the Royal Statistical Society. Series B (Methodological) (1984) 353-388.

[4] M. L. Bujorianu, J. Lygeros, Toward a general theory of stochastic hybrid systems, in: Stochastic Hybrid Systems, Springer, 2006, pp. 3-30.

[5] W. Glover, J. Lygeros, A stochastic hybrid model for air traffic control simulation, in: Hybrid Systems: Computation and Control, Springer, 2004, pp. 372-386.

[6] J. P. Hespanha, Stochastic hybrid systems: Application to communication networks, in: Hybrid systems: computation and control, Springer, 2004, pp. 387-401.

[7] J. Hu, W.-C. Wu, S. Sastry, Modeling subtilin production in bacillus subtilis using stochastic hybrid systems, in: Hybrid Systems: Computation and Control, Springer, 2004, pp. 417-431.

[8] A. R. Teel, A. Subbaraman, A. Sferlazza, Stability analysis for stochastic hybrid systems: a survey, Automatica 50 (10) (2014) 2435-2456. 
[9] A. R. Teel, Stochastic hybrid inclusions with diffusive flows, in: Proceedings of the 53rd IEEE Conference on Decision and Control, 2014, pp. 3071-3076.

[10] R. Goebel, R. G. Sanfelice, A. R. Teel, Hybrid dynamical systems: modeling, stability, and robustness, Princeton University Press, 2012.

[11] C. Cai, R. Goebel, A. R. Teel, Smooth Lyapunov functions for hybrid systems Part II:(pre) asymptotically stable compact sets, IEEE Transactions on Automatic Control 53 (3) (2008) 734-748.

[12] A. Subbaraman, A. R. Teel, On the equivalence between global recurrence and the existence of a smooth Lyapunov function for hybrid systems, Systems \& Control Letters 88 (2016) 54-61.

[13] A. Subbaraman, M. Hartman, A. R. Teel, A stochastic hybrid algorithm for robust global almost sure synchronization on the circle: all-to-all communication, in: Proceedings of the 52nd IEEE Conference on Decision and Control, 2013, pp. 600-605.

[14] S. Venkateswaran, J. T. Isaacs, K. Fregene, R. Ratmansky, B. M. Sadler, J. P. Hespanha, U. Madhow, RF source-seeking by a Micro aerial vehicle using rotation-based angle of arrival estimates, in: Proceedings of the American Control Conference (ACC), IEEE, 2013, pp. 2581-2587.

[15] J. I. Poveda, A. R. Teel, D. Nesic, Flexible nash seeking using stochastic difference inclusions, in: Proceedings of the American Control Conference (ACC), IEEE, 2015, pp. 2236-2241.

[16] S. P. Meyn, R. L. Tweedie, Markov chains and stochastic stability, Cambridge University Press, 2009.

[17] J. T. Isaacs, C. Magee, A. Subbaraman, F. Quitin, K. Fregene, A. R. Teel, U. Madhow, J. P. Hespanha, GPS-optimal micro air vehicle navigation in degraded environments, in: Proceedings of the American Control Conference (ACC), IEEE, 2014, pp. 2581-2587.

[18] A. Subbaraman, A. R. Teel, Robustness of recurrence for a class of stochastic hybrid systems, in: Proceedings of the 5th IFAC Conference on Analysis and Design of Hybrid systems, 2015, pp. 304-309.

[19] A. R. Teel, On sequential compactness of solutions for a class of stochastic hybrid systems, in: Proceedings of the American Control Conference (ACC), IEEE, 2014, pp. 4512-4517.

[20] R. T. Rockafellar, R. J.-B. Wets, Variational analysis, Vol. 317, Springer, 1998.

[21] A. Subbaraman, A. R. Teel, A Krasovskii-LaSalle function based recurrence principle for a class of stochastic hybrid systems, in: 54th IEEE Conference on Decision and Control (Accepted), 2014.

[22] A. R. Teel, On a recurrence principle for a class of stochastic hybrid systems, Proceedings of the American Control Conference (2014) 4518-4523.

[23] A. R. Teel, J. Hespanha, A. Subbaraman, A converse Lyapunov theorem and robustness for asymptotic stability in probability, IEEE Transactions on Automatic Control (2014) 2426-2441. 
[24] A. Abate, S. Amin, M. Prandini, J. Lygeros, S. Sastry, Computational approaches to reachability analysis of stochastic hybrid systems, in: International Workshop on Hybrid Systems: Computation and Control, Springer, 2007, pp. 4-17.

[25] A. Abate, M. Prandini, J. Lygeros, S. Sastry, Probabilistic reachability and safety for controlled discrete time stochastic hybrid systems, Automatica 44 (11) (2008) 2724-2734.

[26] A. Subbaraman, A. R. Teel, A converse Lyapunov theorem for strong global recurrence, Automatica 49 (10) (2013) 2963-2974.

[27] R. Goebel, A. R. Teel, Solutions to hybrid inclusions via set and graphical convergence with stability theory applications, Automatica 42 (4) (2006) 573-587.

[28] A. Subbaraman, Robust stability theory for stochastic dynamical systems, PhD Thesis, 2016. 